\title{
Unified View of The Local Cation Ordered State in Inverse Spinel Oxides
}

Jue Liu ${ }^{\ddagger}, *$ Xuelong Wang ${ }^{\S, \#}$, Olaf J. Borkiewicz", Enyuan $\mathrm{Hu}^{\S}$, Rui-Juan Xiao\#, Liquan Chen\#, Katharine Paget,**

Neutron Scattering Division, Oak Ridge National Laboratory, Oak Ridge, Tennessee, 37831, United States

${ }^{\S}$ Chemistry Division, Brookhaven National Laboratory, Upton, New York, 11973, United States \# Institute of Physics, Chinese Academy of Sciences, 100190 Beijing, China

" X-ray Science Division, Advanced Photon Source, Argonne National Laboratory, Argonne, Illinois 60439, United States
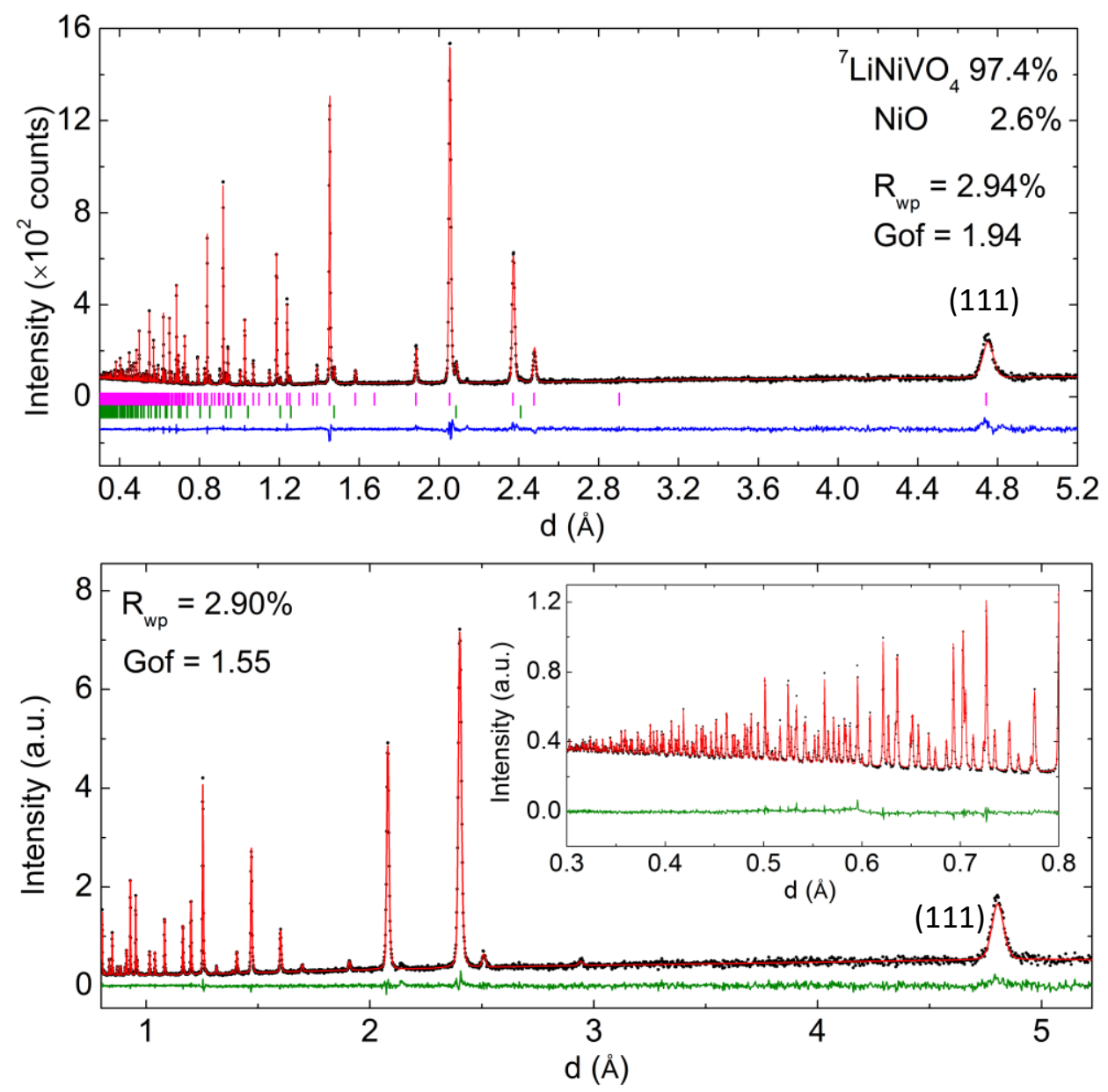

Figure S1. Neutron diffraction patterns of $\mathrm{V}^{7} \mathrm{LiNi}^{\mathrm{O}} \mathrm{O}_{4}$ and ${ }^{7} \mathrm{Li}[\mathrm{CrTi}] \mathrm{O}_{4}$. (111) peak is labeled for easy comparison. 

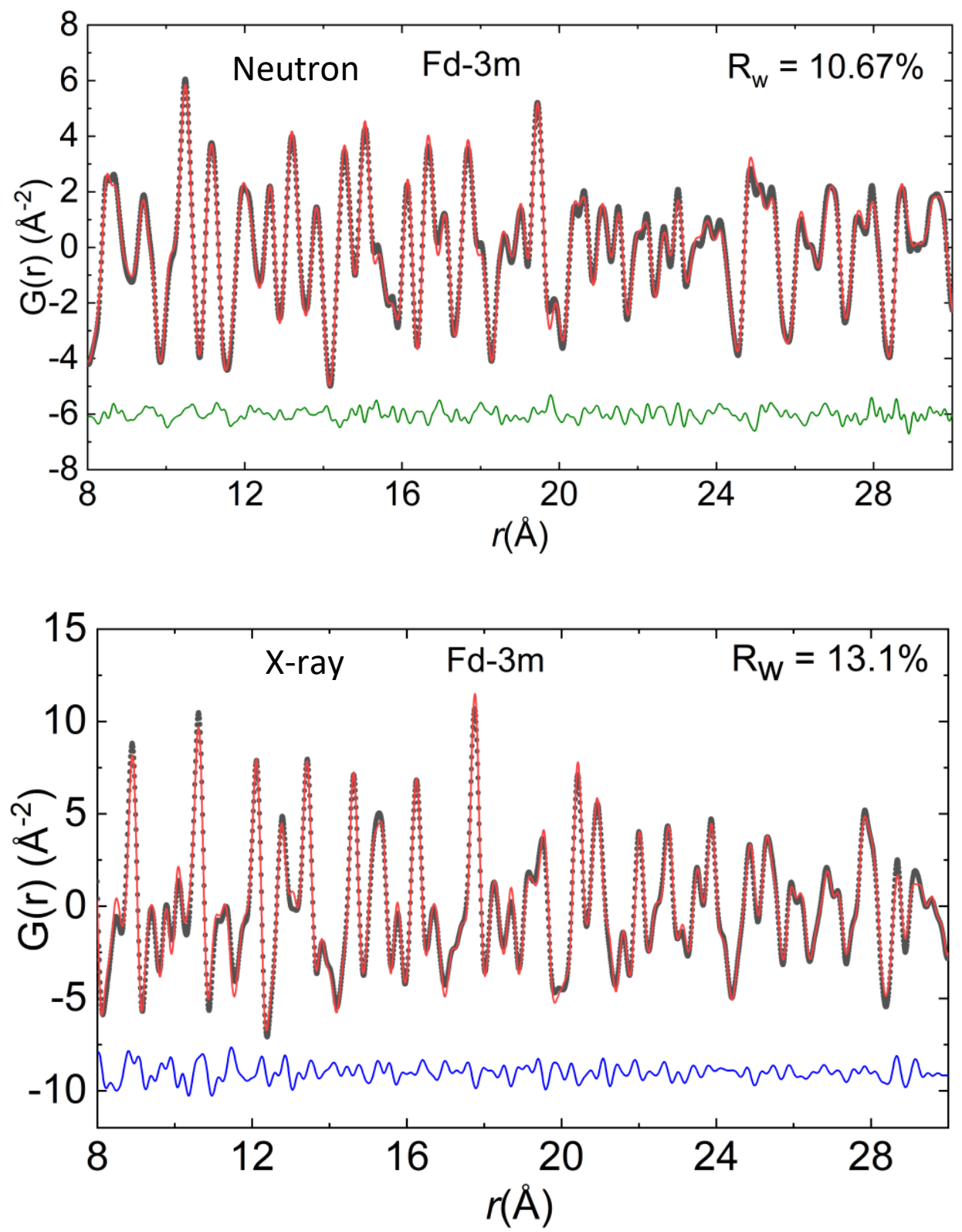

Figure S2. Least square refinements of the intermediate range cation disordered structure (S.G. $\mathrm{Fd}-3 \mathrm{~m})$ of ${ }^{7} \mathrm{LiNiVO}_{4}$ using neutron and X-ray PDF data (8 to $30 \AA$ ). 


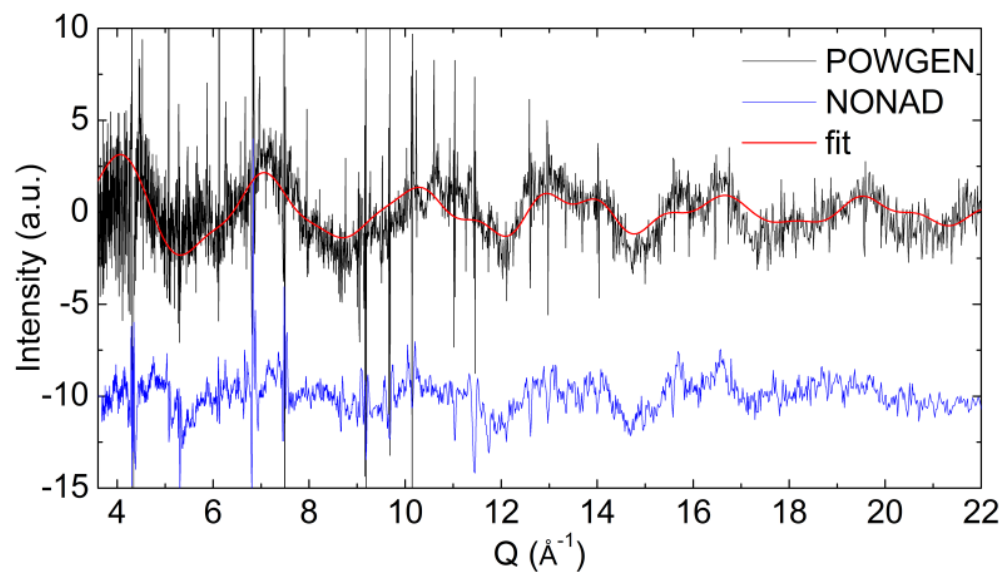

Figure S3. Modeling of the diffuse scattering signal of ${ }^{7} \mathrm{LiNiVO}_{4}$ using cation ordered model up to the second metal-metal shell.
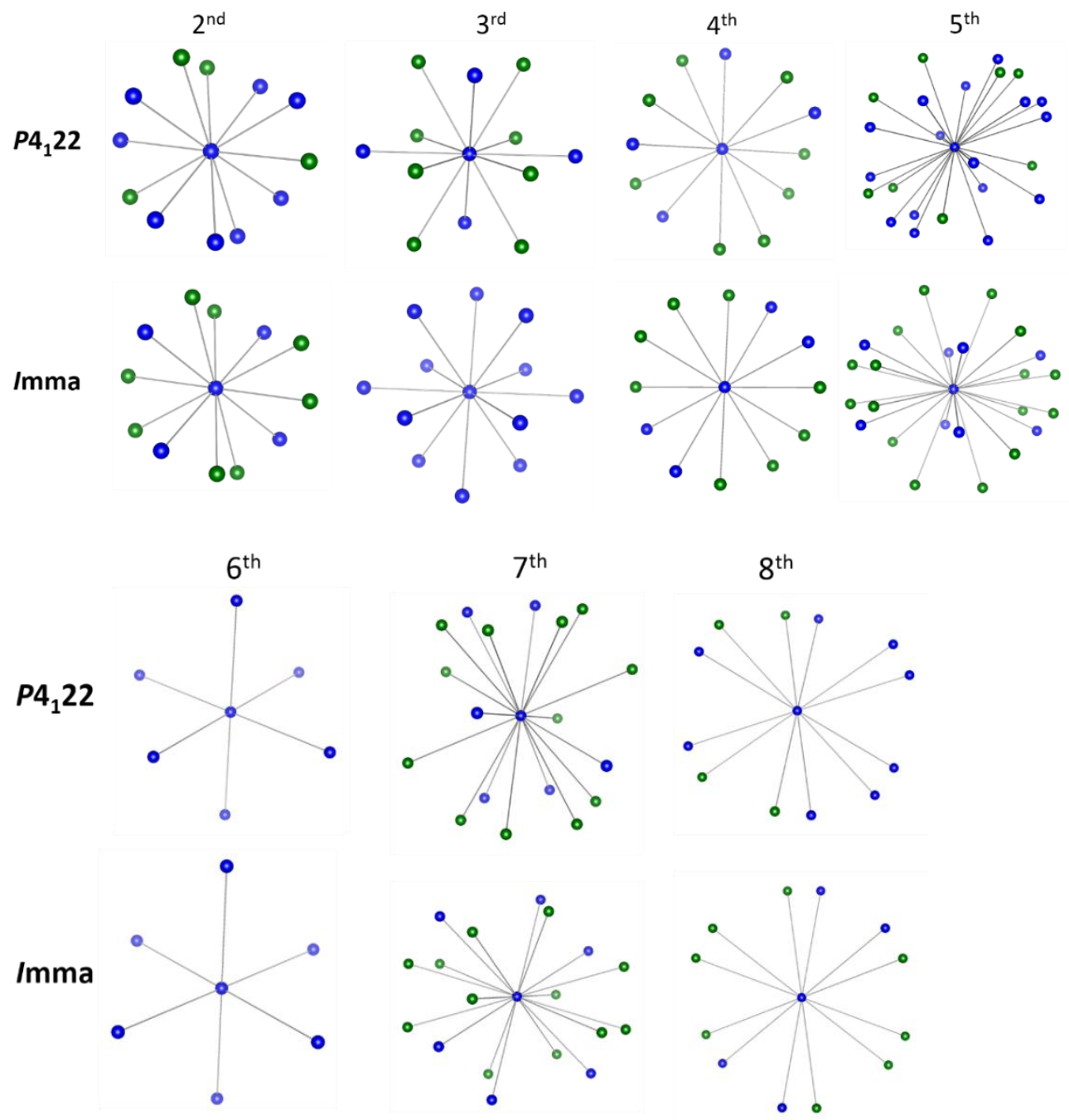

Figure S4. The higher order cation shells with sphere expansion (up to the $8^{\text {th }}$ ) for inverse spinel $\mathrm{V}[\mathrm{BC}] \mathrm{O}_{4}$ with $\mathrm{P}_{4} 22$ or Imma ordered structures. 

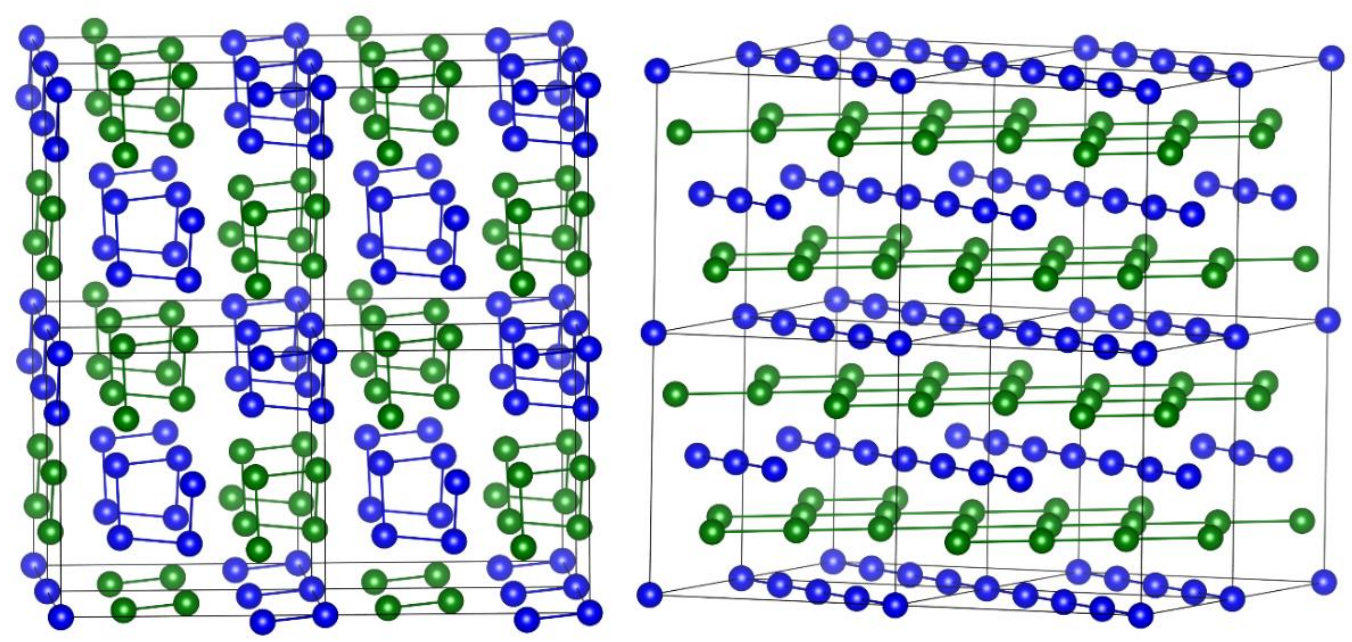

Figure S5. The expanded $2 \times 2 \times 2$ cubes for the inverse spinel V[BC]O $\mathrm{O}_{4}$ with $P 4_{1} 22$ (left) or Imma (right) ordered structures. It is worth noting that when using Evjen method to calculate the electrostatic repulsive energy using the cube expansion, ${ }^{1}$ the weights of $1 / 2,1 / 4$ and $1 / 8$ are given for the cations situated on a face, an edge and a corner of the cube.

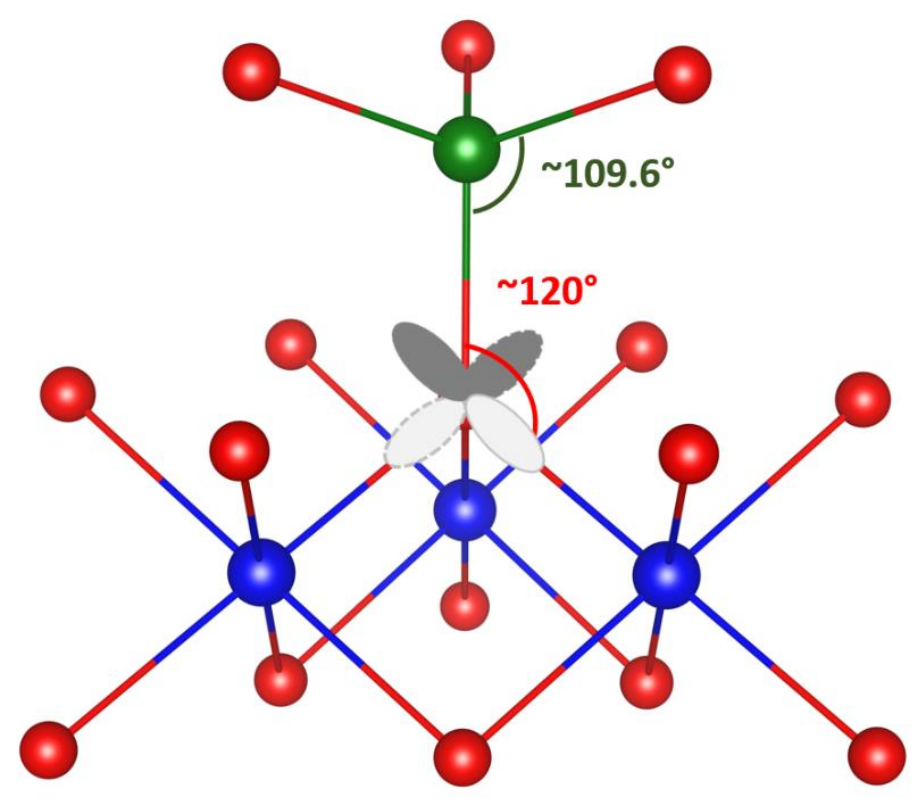

Figure S6. Illustration of the orientation of oxygen $2 p$ orbitals surrounding the octahedral and tetrahedral sites (only two out of three oxygen $2 \mathrm{p}$ orbitals are shown for the octahedral type overlap for clarity). Since the three oxygen $2 p$ orbitals $\left(p_{x}, p_{y}\right.$ and $p_{z}$ ) are mutual, there is only one type of effective covalent overlapping between oxygen $2 p$ orbitals and TM d orbitals (either with octahedral cation or tetrahedral cation) due to the angle mismatch $\left(\mathrm{TM}_{\mathrm{oct}}-\mathrm{O}-\mathrm{TM}_{\mathrm{tet}} \sim 120^{\circ}\right)$. 

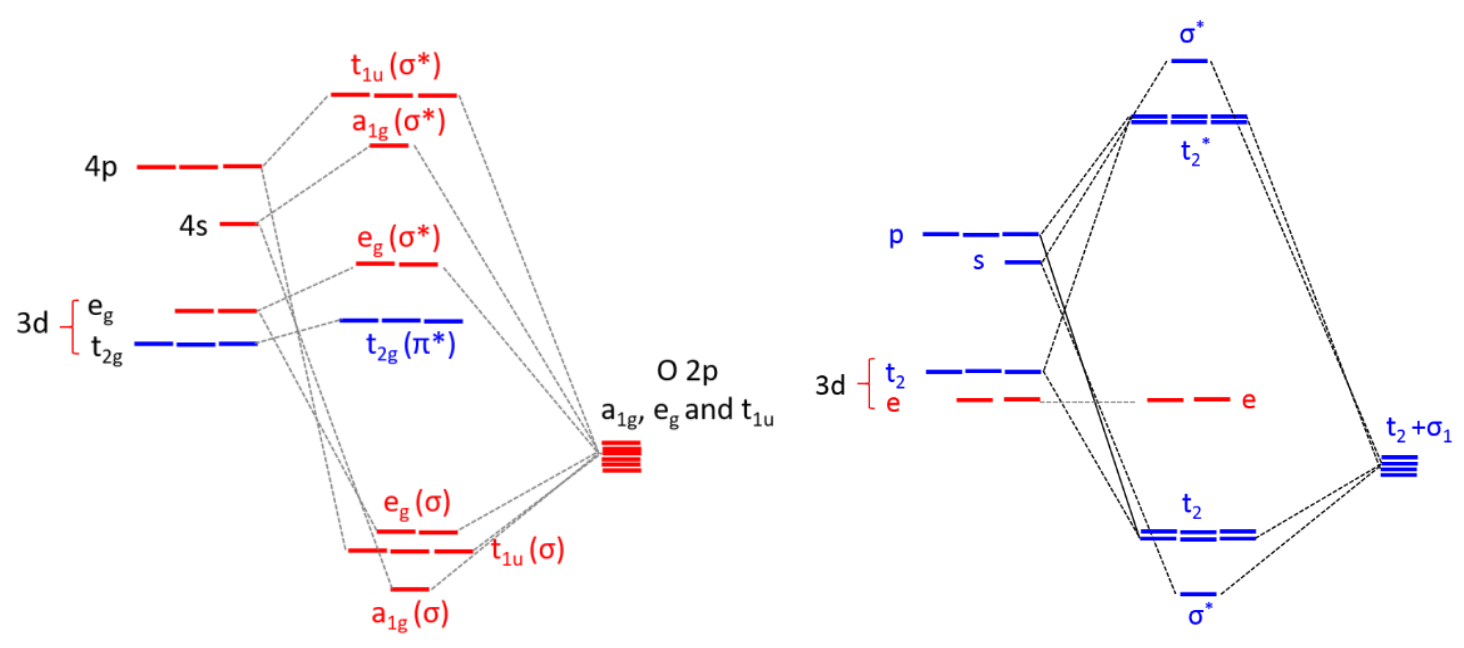

Figure S7. Approximate molecular orbitals of TM cations ( $3 \mathrm{~d}$ here) in the octahedral (left) and tetrahedral (right) site in spinel oxides.

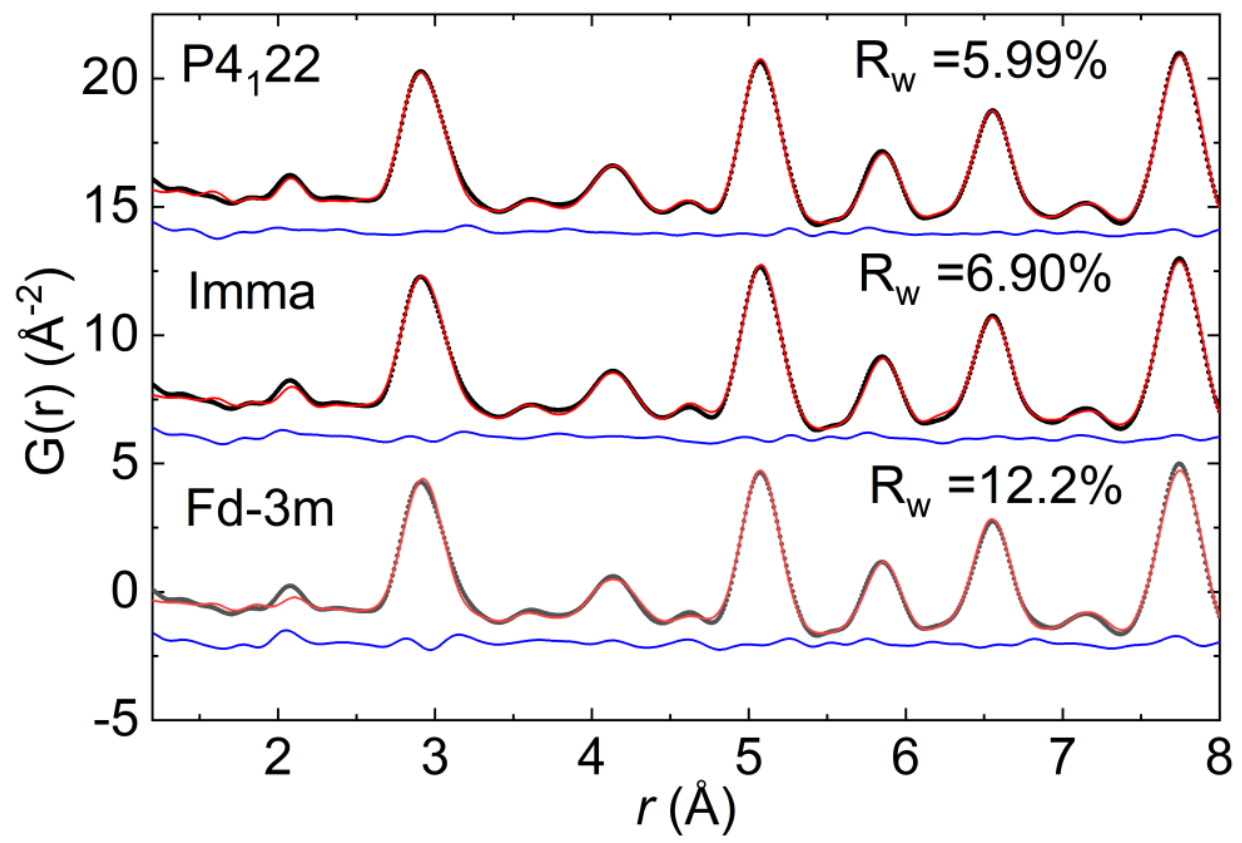

Figure S8. Local structure refinement of ${ }^{7} \mathrm{LiCoVO}_{4}$ using neutron PDF (1-8 ̊). Three different local structure models are used: the cation disorder $F \mathrm{~d}-3 \mathrm{~m}$, orthorhombic ordered Imma and tetragonal ordered $P 4_{1} 22$. 

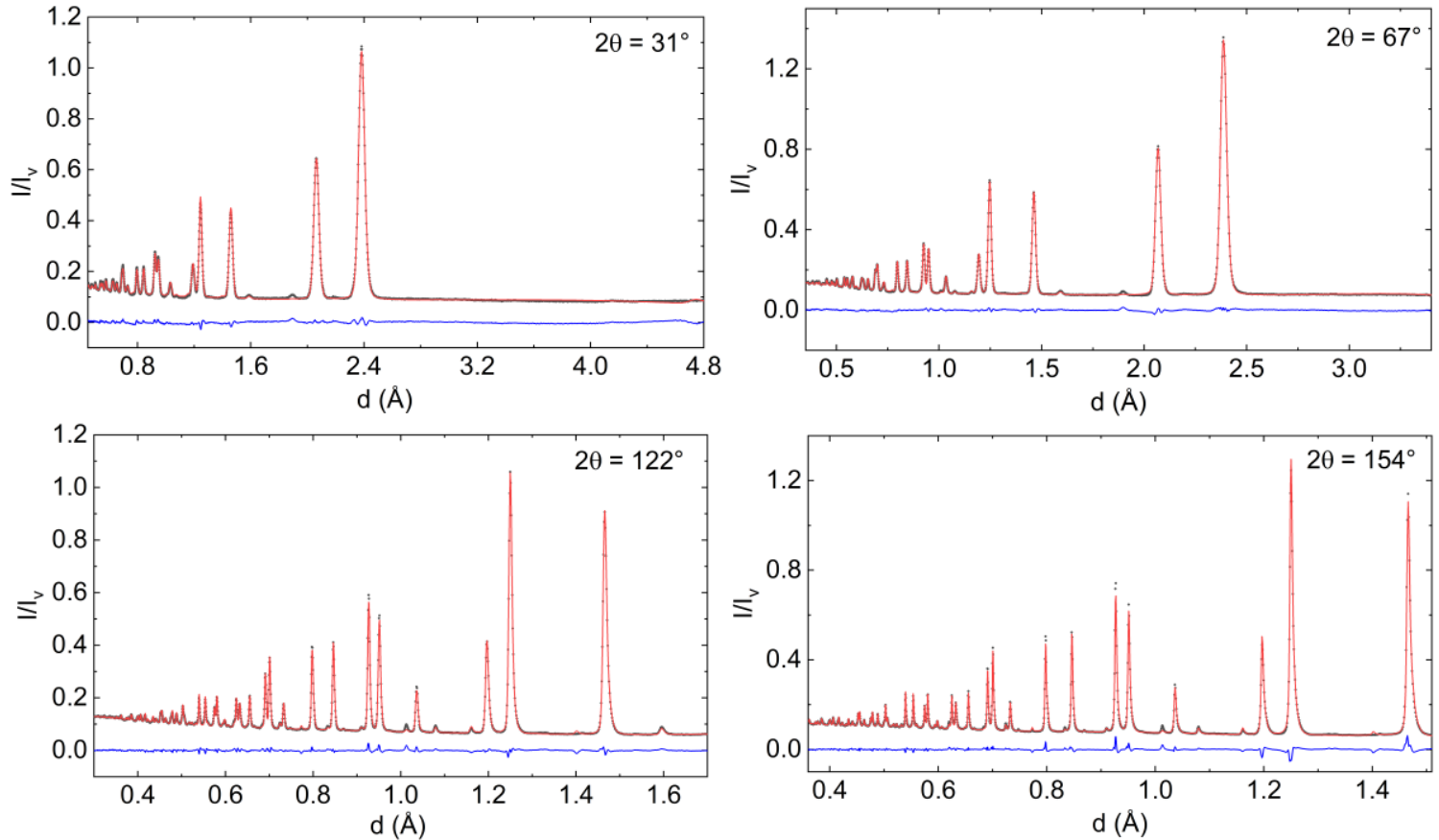

Figure S9. Rietveld refinement of the structure of ${ }^{7} \mathrm{LiCoVO}_{4}$ against neutron diffraction data (NOMAD) using the cation disordered Fd-3m model.
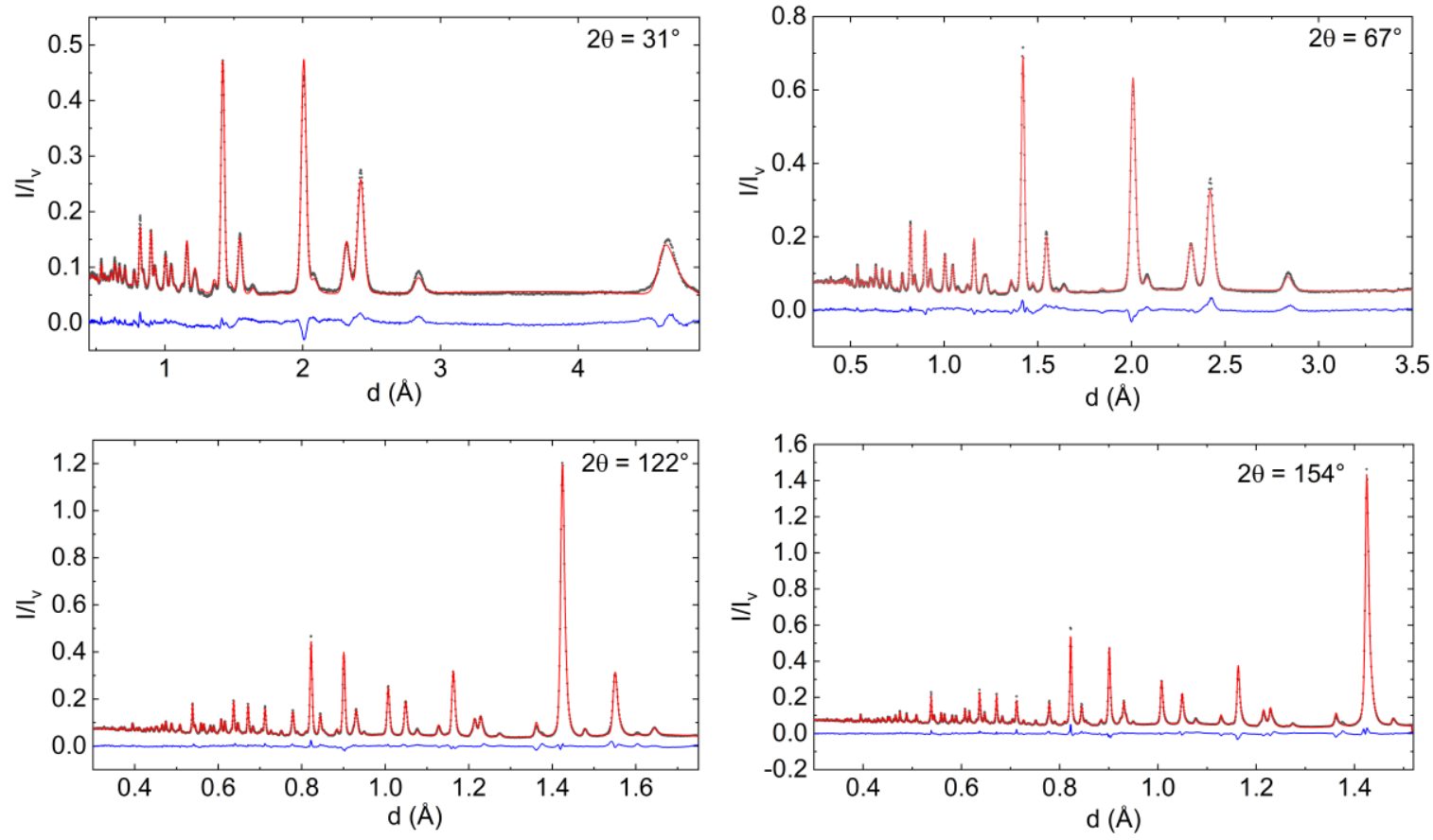

Figure S10. Rietveld refinement of the cation disordered structures (S.G. Fd-3m) of $\mathrm{NiAl}_{2} \mathrm{O}_{4}$ using neutron diffraction data (NOMAD). 


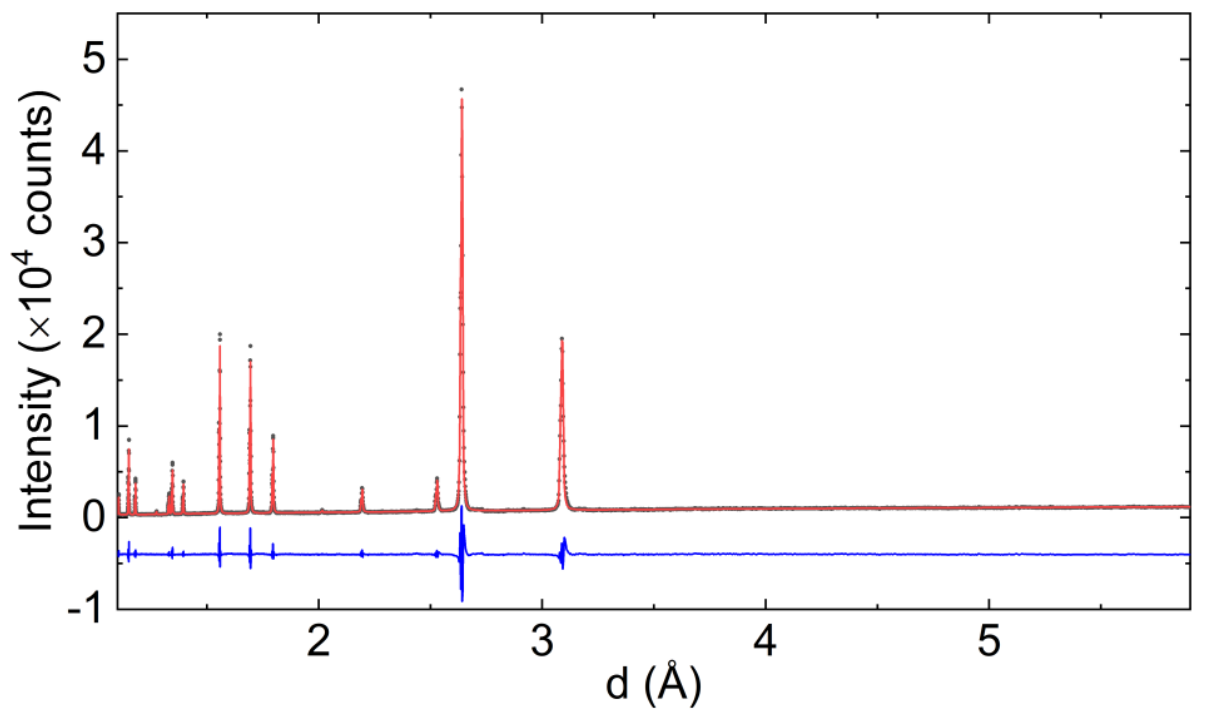

Figure S11. Rietveld refinement of the cation disordered structures (S.G. Fd-3m) of $\mathrm{MgIn}_{2} \mathrm{O}_{4}$ using XRD ( $\mathrm{Cu} \mathrm{K} \alpha$ radiation).
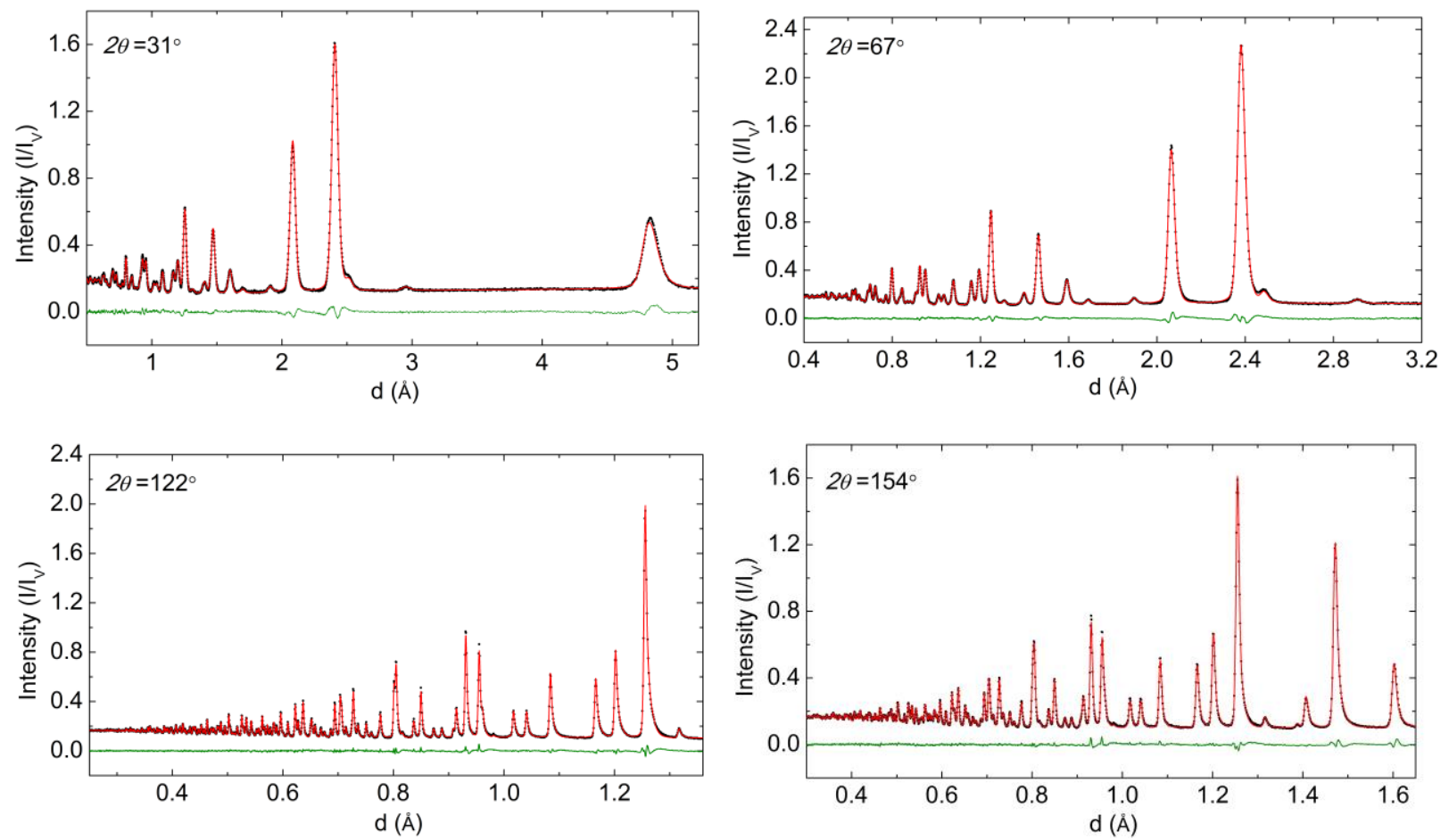

Figure S12. Rietveld refinement of the structure of ${ }^{7} \mathrm{LiCrTiO}_{4}$ using the cation disordered $F \mathrm{~d}-3 \mathrm{~m}$ model (data collected from NOMAD, SNS). 


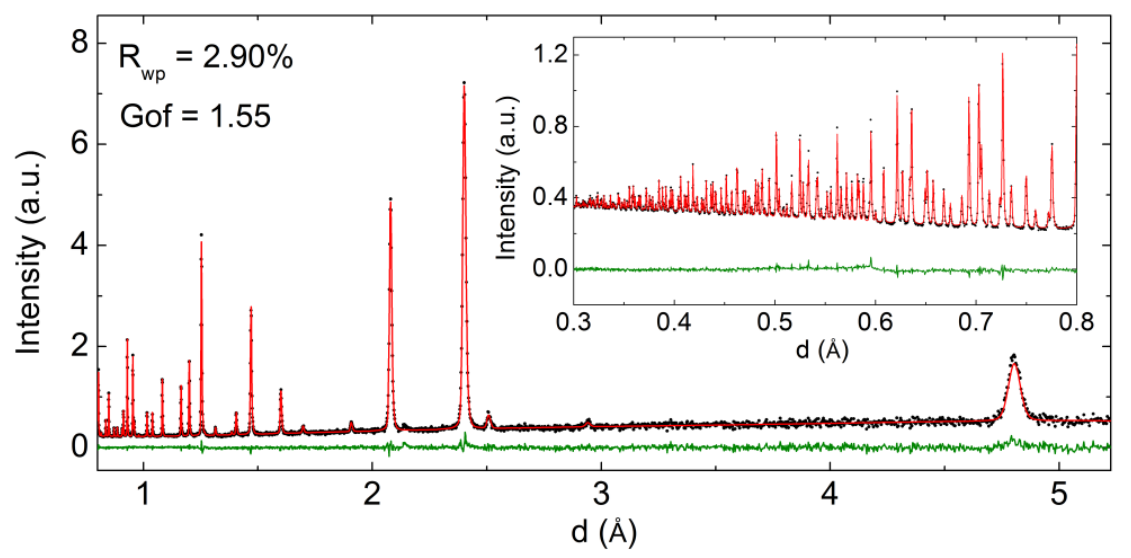

Figure S13. Rietveld refinement of the structure of ${ }^{7} \mathrm{LiCrTiO}_{4}$ using the cation disordered $F \mathrm{~d}-3 \mathrm{~m}$ model (data collected from POWGEN, SNS).

(a)

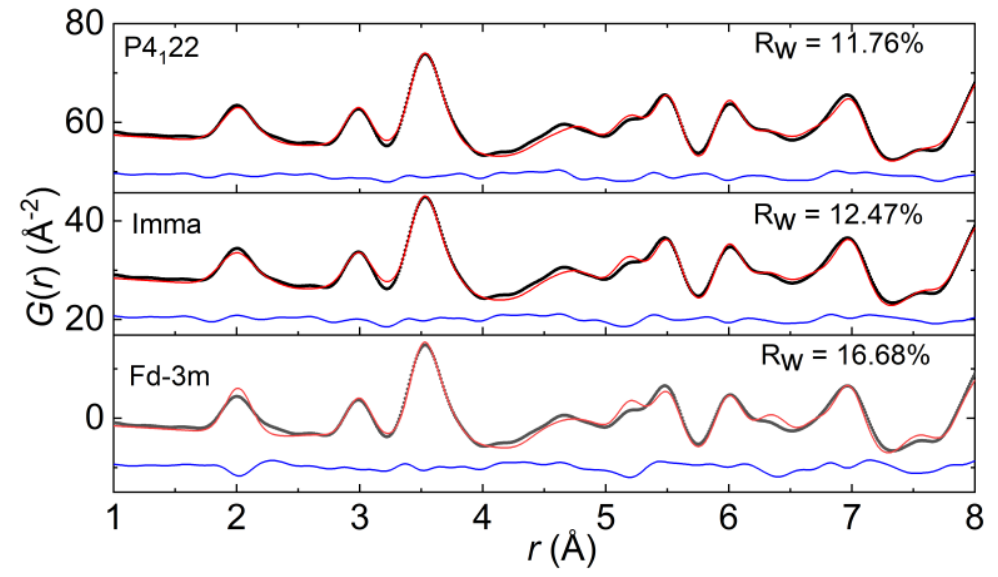

(b)

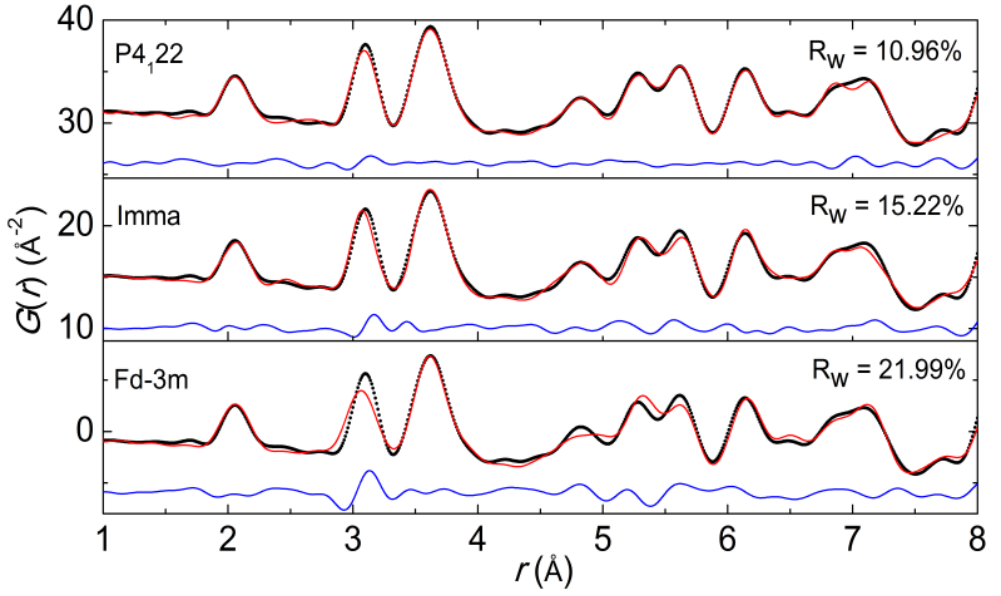

Figure S14. Least square refinements of the three local structure models of $\mathrm{Zn}_{2} \mathrm{TiO}_{4}$ (a) and $\mathrm{Zn}_{2} \mathrm{SnO}_{4}$ (b) using X-ray PDF (11-ID-B, APS, 1-8 ̊). 


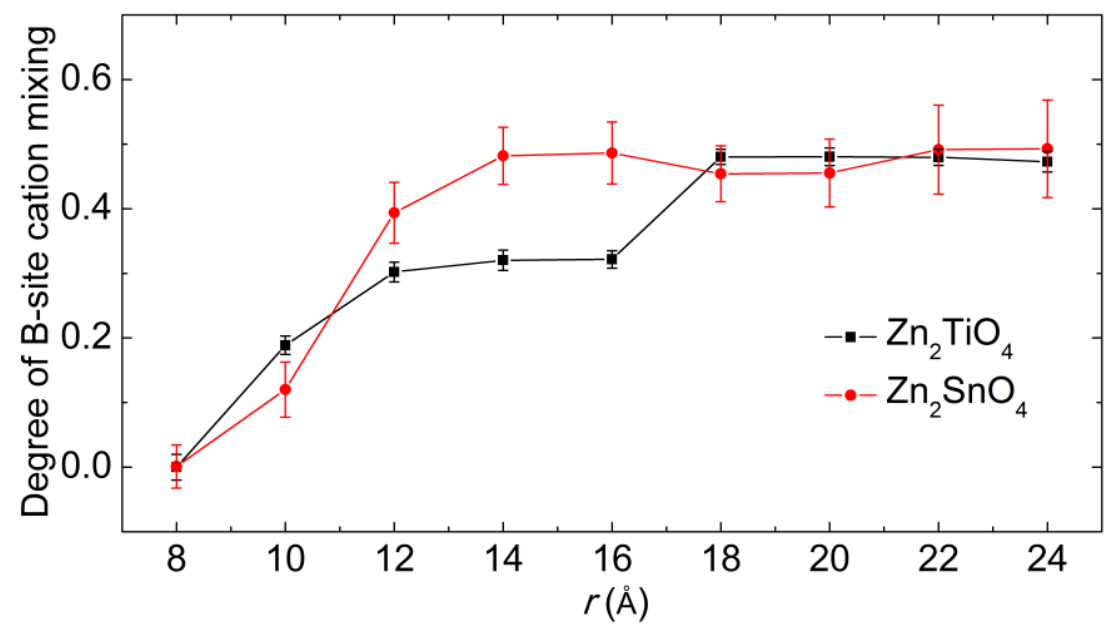

Figure S15. The degrees of $\mathrm{B}$-site cation ordering ( $\mathrm{Zn}-\mathrm{Ti}$ and $\mathrm{Zn}-\mathrm{Sn})$ as a function of atomic pair distances. Box-car type fits were carried out with an interval of $8 \AA$ up to $24 \AA$. Neutron PDF (NOMAD, SNS) and X-ray PDF (11-ID-B, APS) were used for the structure refinement of $\mathrm{Zn}_{2} \mathrm{TiO}_{4}$ and $\mathrm{Zn}_{2} \mathrm{SnO}_{4}$ separately. The degree of $\mathrm{B}$-site cation ordering shall be $50 \%$ for the cation disordered structure.
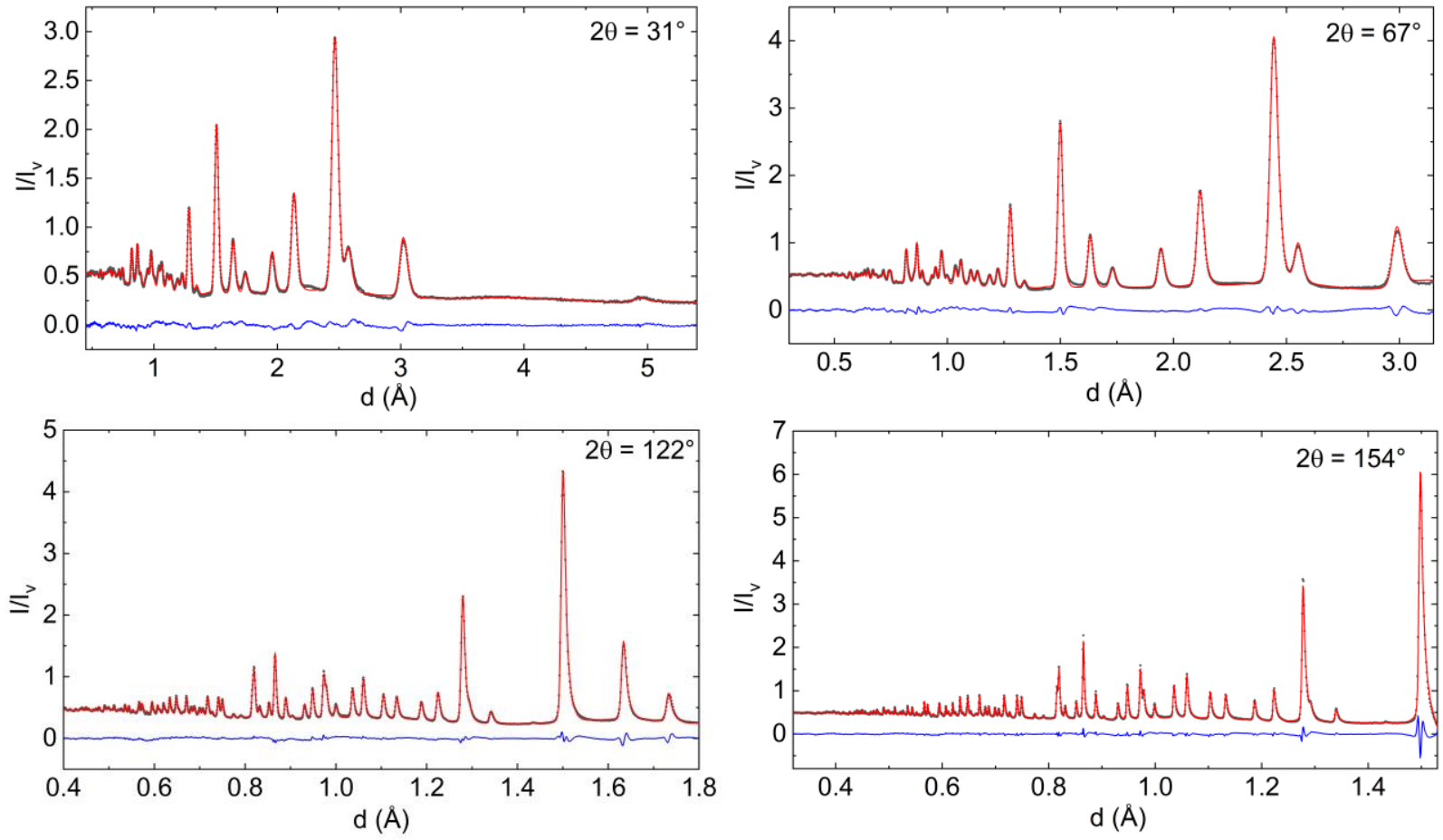

Figure 16. Rietveld refinement of the cation disordered long-range structure (S.G. Fd-3m) of $\mathrm{Zn}_{2} \mathrm{TiO}_{4}$ using neutron diffraction data 

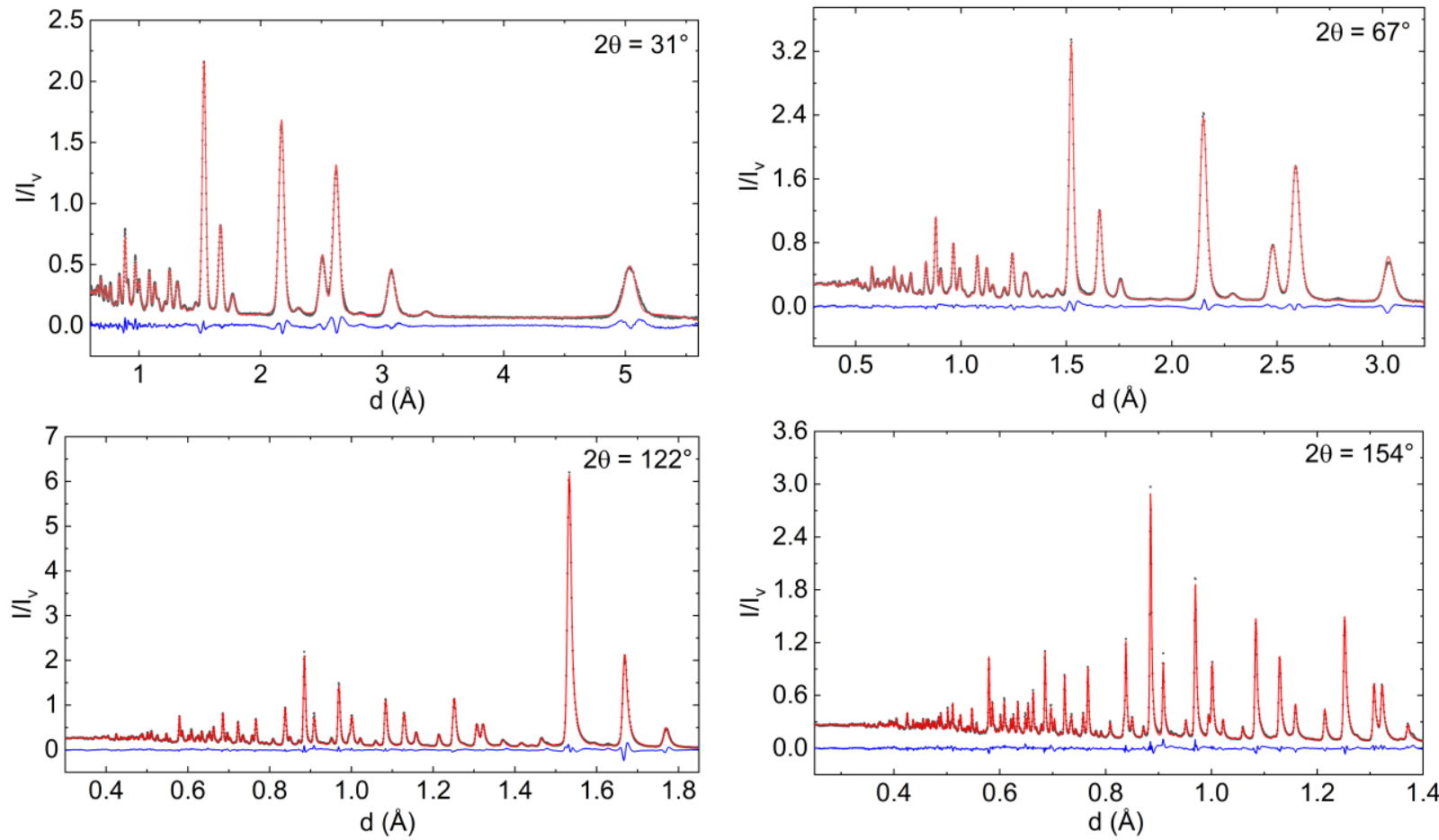

Figure S17. Rietveld refinement of the cation disordered long-range structure (S.G.Fd-3m) of $\mathrm{Zn}_{2} \mathrm{SnO}_{4}$ using neutron diffraction data.

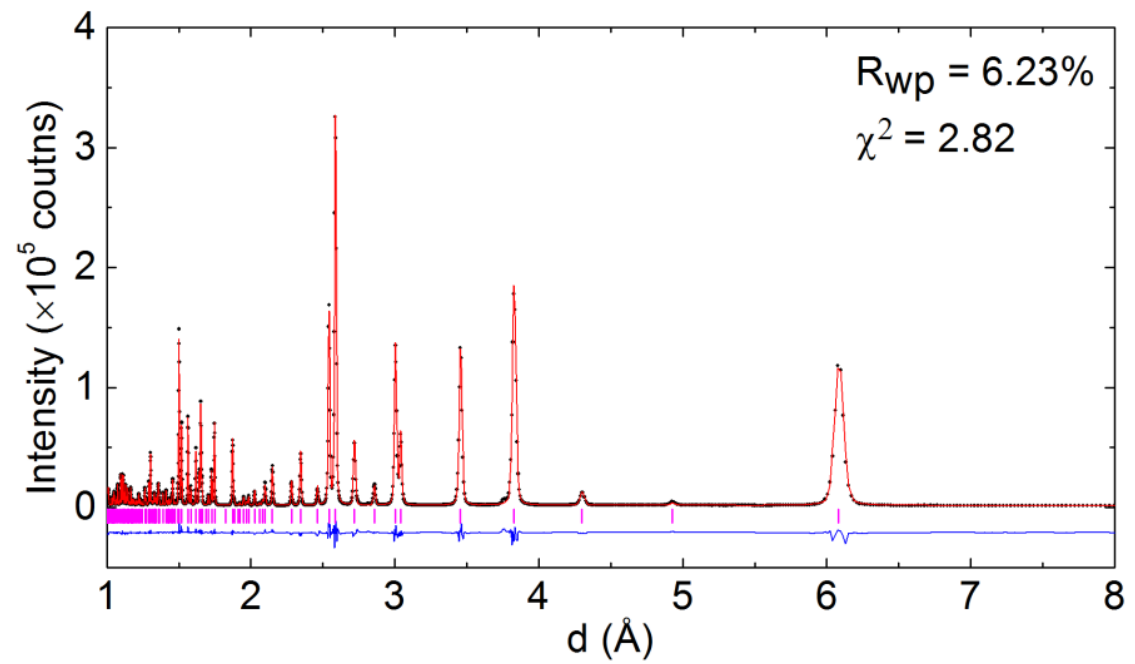

Figure S18. Rietveld refinement of the long-range structure (S.G. P4 $4_{1} 22$ ) of $\mathrm{LiZnNbO}_{4}$ using (a) synchrotron $\mathrm{XRD}(\lambda=0.2113 \AA)$ and (b) neutron diffraction data (POWGEN). 


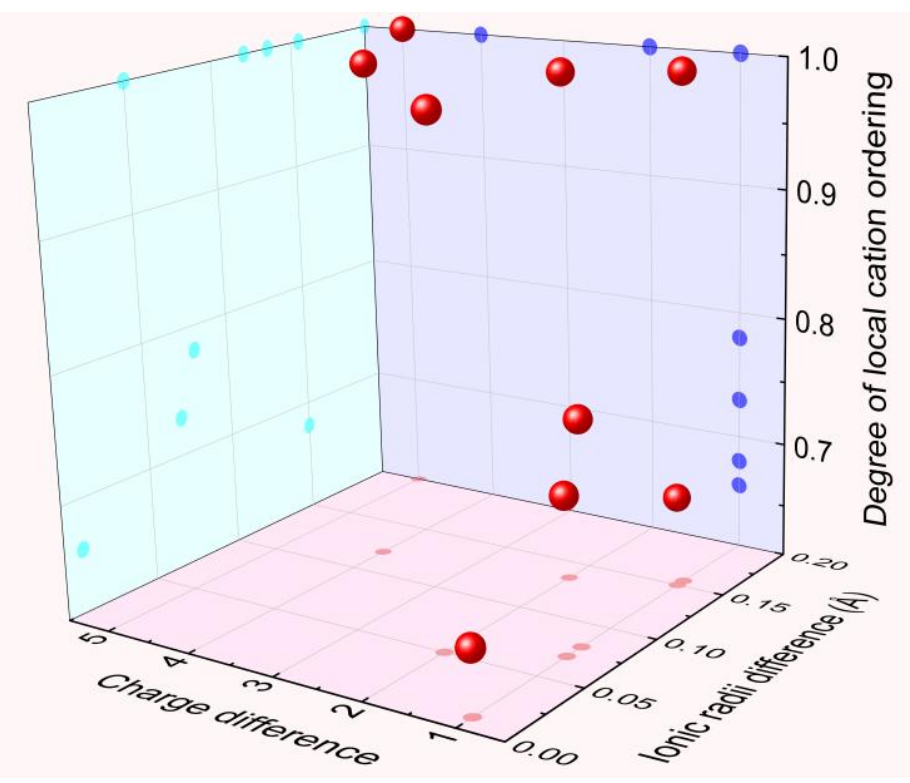

Figure S19. The relationship between the degree of local cation ordering and the charge/ionic radii difference for inverse spinel oxides with highly ionic lattice. The combination of any two parameters, i.e. charge-ionic radii difference, ionic radii difference-degree of local cation ordering, charge difference-degree of local cation ordering, are projected into corresponding planes for clarity.
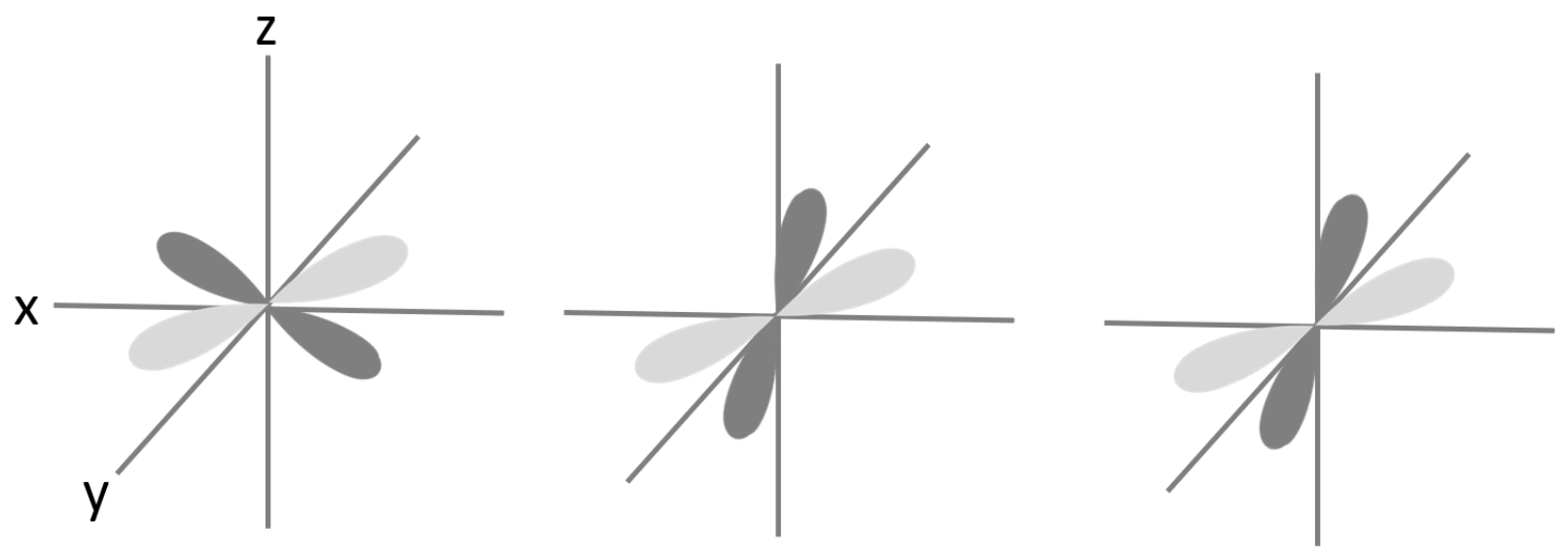

Figure S20. Illustration of the orientation of $t_{2 g}$ orbitals with ligand oxygen in the cubic crystal field (octahedral environment). 


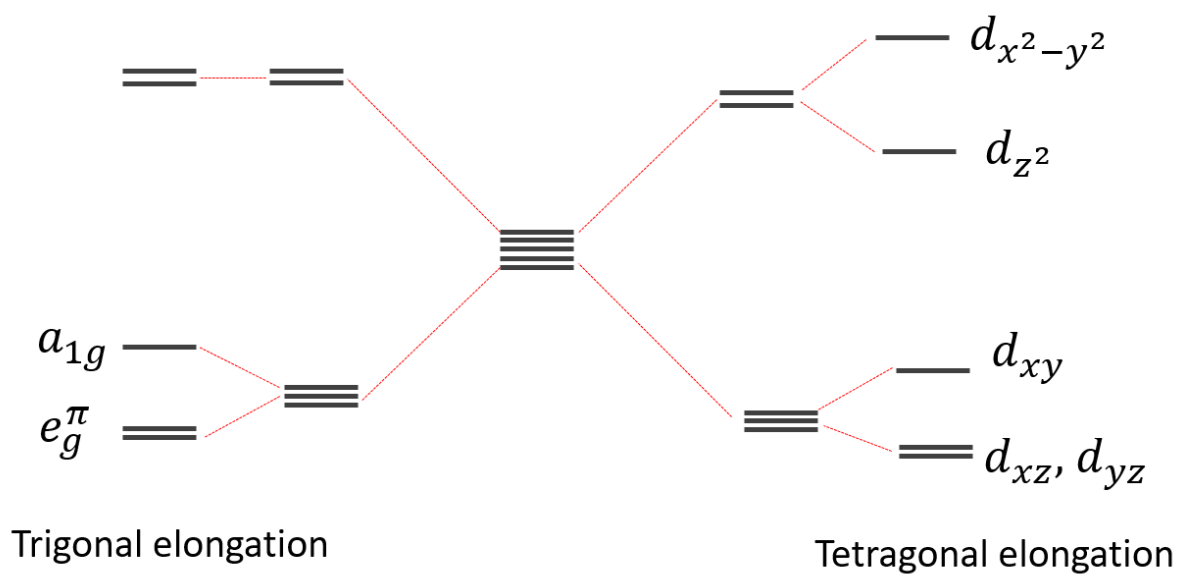

Figure S21. The two plausible (tetragonal and trigonal) distortion of $t_{2 g}$ orbitals of transition metal on the octahedral site. Only the elongation situation is shown here, the distortion can also be of contraction type.

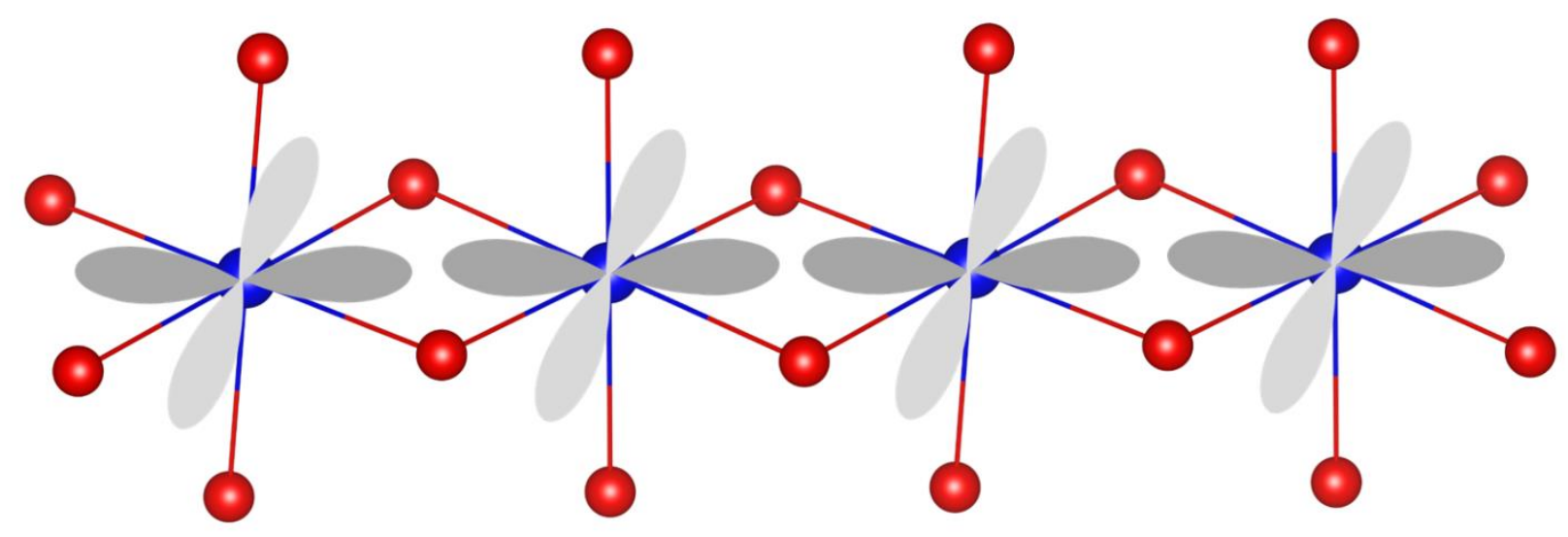

Figure S22. The potential direct $t_{2 g}-t_{2 g}$ orbital overlapping of octahedral (B-site) cations in the spinel oxides.

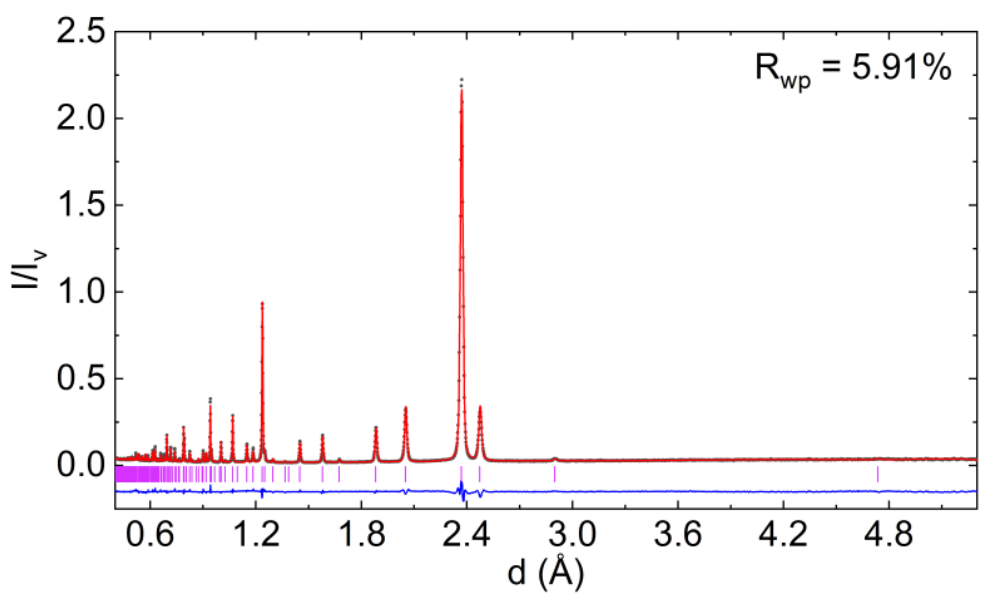


Figure S23. Rietveld refinement of the cation disordered long-range structure (S.G. Fd-3m) of $\mathrm{LiMn}_{2} \mathrm{O}_{4}$ using $300 \mathrm{~K}$ neutron diffraction data (POWGEN).
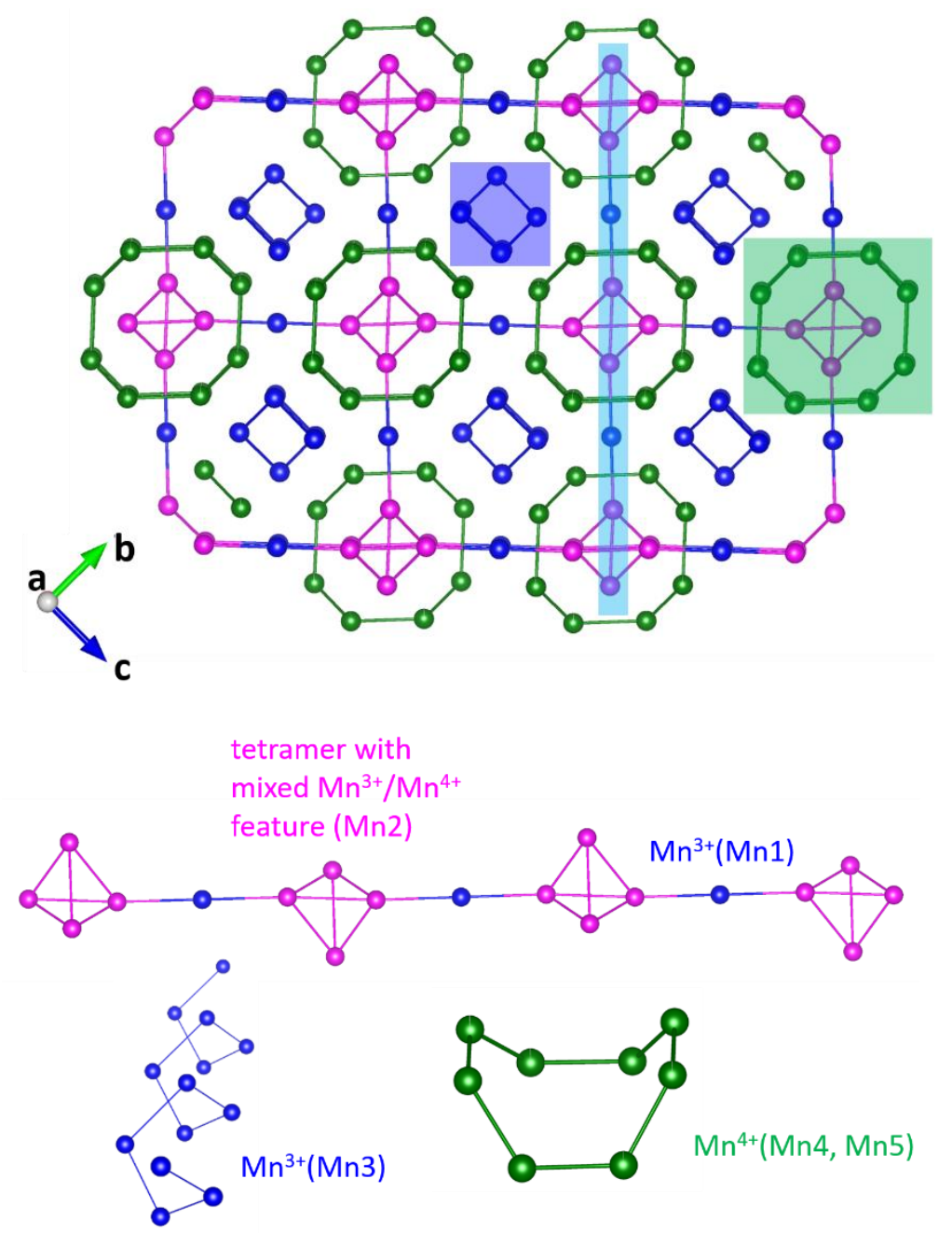

Figure S24. The illustration of the connection of different $\mathrm{Mn}^{3+}$ and $\mathrm{Mn}^{4+}$ environments in charge ordered structure of $\mathrm{LiMn}_{2} \mathrm{O}_{4}$.

\section{Equation S1}

For ternary spinel oxides $\mathrm{A}[\mathrm{BC}] \mathrm{O}_{4}$, the structure factor for the 111 reflection $\left(\mathrm{F}_{111}\right)$ is:

$$
\begin{gathered}
\mathrm{F}_{111}=\sum_{n} f_{A} e^{2 \pi i\left(x_{n}+y_{n}+z_{n}\right)}+\left(\sum_{m} f_{B} e^{2 \pi i\left(x_{m}+y_{m}+z_{m}\right)}+\sum_{m} f_{A} e^{2 \pi i\left(x_{m}+y_{m}+z_{m}\right)}\right)+\sum_{S} f_{o} e^{2 \pi i\left(x_{O}+y_{O}+z_{o}\right)}= \\
4 \sqrt{2} f_{A}-4\left(f_{\mathrm{B}}+f_{\mathrm{C}}\right)+8 f_{o}\left(\cos 6 \pi x_{O}-3 \cos 2 \pi x_{O}\right)
\end{gathered}
$$

Where $f_{A}, f_{B}, f_{C}$ and $f_{O}$ are the atomic form factors for X-ray scattering or nuclear scattering lengths for neutron scattering, $x_{0}$ is the atomic position for the oxygen ion on $32 \mathrm{e}$ site. Since X-ray form factor $(f o)$ is relative small. Thus, the structure factor for the 111 reflection can be approximated as following for the Xray scattering:

$$
\mathrm{F}_{111}=4 \sqrt{2} f_{A}-4\left(f_{\mathrm{B}}+f_{\mathrm{C}}\right)
$$


If $f_{A} \ll f_{B}$ or $f_{C}$, as the case for $\mathrm{X}$-ray scattering for $\mathrm{Li}[\mathrm{BC}] \mathrm{O}_{4}$ spinel, $\left|F_{111}\right|^{2} \sim 16\left(f_{\mathrm{B}}+f_{\mathrm{C}}\right)^{2}$. Thus, the 111 reflection shall be very strong.

In contrast, if $f_{A} \sim f_{B}$ or $f_{C}$, as the case for X-ray scattering for $\mathrm{V}[\mathrm{LiTM}] \mathrm{O}_{4}(\mathrm{TM}$ represents $3 \mathrm{~d}$ transition metal), then $\left|F_{111}\right|^{2} \sim f_{C}^{2}$. Thus, the 111 reflection shall be much weaker relative to that from the $\mathrm{Li}[\mathrm{VTM}] \mathrm{O}_{4}$ configuration.

\section{Equation S2}

$$
U_{\text {ioinic }}=-A_{M} \frac{Z_{A} Z_{B} e^{2}}{r}+B_{M} e^{-\frac{r}{\rho}}-C_{w} \frac{1}{r^{6}}+D_{z} h \mu_{v}
$$

Where the first term represents the contribution from coulombic potential energy, the second term represents the contribution from Born-Mayer repulsive energy, the third term is from the Van der Waals attractive force (dipole-dipole interaction between ions), and the last term is the zero point energy (from the vibrational modes).The energy contribution from the last two terms is very limited and has opposite sign (cancel each other), thus, they will not be included in the current discussion.

$\mathrm{A}_{\mathrm{M}}$ represents the Madelung constant, $\mathrm{B}_{\mathrm{M}}$ represents the Born-Mayer constant, $\mathrm{C}_{\mathrm{w}}$ represents the Van der Waals constant and $\mathrm{D}_{\mathrm{z}}$ is 2.25 .

\section{Equation S3}

$$
\begin{aligned}
\Delta E & =U_{\text {ionic1 } 1}-U_{\text {ionic } 2}=E_{\text {config } 1}-E_{B_{\text {config } 2}}=-A_{M} e^{2}\left(\sum_{i, j}^{c o n f i g 2} \frac{Z_{i} Z_{j}}{r_{i j}}-\sum_{i, j}^{\text {config } 1} \frac{Z_{i} Z_{j}}{r_{i j}}\right) \\
& =-A_{M} e^{2} \sum_{n} \frac{\sum_{i j} Z_{i} Z_{j}-\sum_{s m} Z_{s} Z_{m}}{r_{n}}
\end{aligned}
$$

Where $\mathrm{A}_{\mathrm{M}}$ represents the Madelung constant for the spinel oxide lattice, $r_{n}$ represents the $n$th cation shell.

\begin{tabular}{|c|c|c|c|c|c|c|c|c|c|}
\hline \multirow{2}{*}{$\begin{array}{c}\text { Lattice } \\
\text { Parameters }\end{array}$} & \multicolumn{3}{|c|}{$F \mathrm{~d}-3 \mathrm{~m}$} & \multicolumn{3}{|c|}{$P 4122$} & \multicolumn{3}{|c|}{ Imma } \\
\hline & refinement & DFT & diff. & refinement & DFT & diff. & refinement & DFT & diff. \\
\hline $\mathrm{a}$ & 8.20992 & 8.26717 & $0.70 \%$ & 5.804 & 5.8387 & $0.60 \%$ & 5.791 & 5.81059 & $0.34 \%$ \\
\hline $\mathrm{b}$ & 8.20992 & 8.28608 & $0.93 \%$ & 5.804 & 5.8387 & $0.60 \%$ & 5.818 & 5.89599 & $1.34 \%$ \\
\hline $\mathrm{c}$ & 8.20992 & 8.27097 & $0.74 \%$ & 8.283 & 8.29088 & $0.095 \%$ & 8.286 & 8.23462 & $\begin{array}{c}- \\
0.62 \%\end{array}$ \\
\hline$\alpha$ & $90^{\circ}$ & 89.9666 & $\begin{array}{c}- \\
0.037 \%\end{array}$ & $90^{\circ}$ & $90^{\circ}$ & 0 & $90^{\circ}$ & $90^{\circ}$ & 0 \\
\hline$\beta$ & $90^{\circ}$ & 90.2115 & $0.24 \%$ & $90^{\circ}$ & $90^{\circ}$ & 0 & $90^{\circ}$ & $90^{\circ}$ & 0 \\
\hline
\end{tabular}

Table S1. Lattice parameters of $\mathrm{LiNiVO}_{4}$ obtained by Neutron/X-ray PDF refinement and DFT calculations. 


\begin{tabular}{|c|c|c|c|c|c|c|c|c|c|}
\hline$\gamma$ & $90^{\circ}$ & 90.2906 & $0.32 \%$ & $90^{\circ}$ & $90^{\circ}$ & 0 & $90^{\circ}$ & $90^{\circ}$ & 0 \\
\hline
\end{tabular}

Table S2. Refined long range structure (Fd-3m) of LiNiVO 4 (POWGEN, SNS)

\begin{tabular}{|c|c|c|c|c|c|c|}
\hline & \multicolumn{3}{|c|}{ S.G. Fd-3m a $=8.21247(3) \AA$} & \multicolumn{2}{|c|}{$\mathrm{R}_{\mathrm{wp}}=2.94 \% \chi^{2}=1.94$} & \\
\hline Atom & Wyck. & $x$ & $y$ & $z$ & Occ. & $B_{\text {iso }}\left(\AA^{2}\right)$ \\
\hline $\mathbf{V}$ & $8 b$ & $3 / 8$ & $3 / 8$ & $3 / 8$ & 1 & $0.34^{*}$ \\
\hline${ }^{7} \mathbf{L i}$ & $16 \mathrm{c}$ & 0 & 0 & 0 & $0.49(1)$ & $0.83(18)$ \\
\hline $\mathbf{N i}$ & $16 \mathrm{c}$ & 0 & 0 & 0 & $0.51(1)$ & $0.24(5)$ \\
\hline $\mathbf{O}$ & $32 \mathrm{e}$ & 0.252 & $0.2529(1)$ & $0.2529(1)$ & 1 & $0.56(1)$ \\
\hline
\end{tabular}

${ }^{*}$ Fixed to the value refined from XRD.

Table S3. Refined long range structure (Fd-3m) of $\operatorname{LiNiVO}_{4}(\lambda=0.2114 \AA$, $11-\mathrm{ID}-\mathrm{B}, \mathrm{APS})$

\begin{tabular}{llccllll}
\hline \multicolumn{6}{c}{ S.G. $F d-3 \mathrm{~m}$} & $\mathrm{a}=8.2193(1) \AA$ & \multicolumn{4}{l}{$\mathrm{R}_{\mathrm{wp}}=4.57 \% \chi^{2}=2.07$} \\
\hline Atom & Wyck. & $x$ & $y$ & $z$ & Occ. & $B_{\text {iso }}\left(\AA^{2}\right)$ \\
$\mathbf{V}$ & $8 \mathrm{~b}$ & $3 / 8$ & $3 / 8$ & $3 / 8$ & 1 & $0.34(3)$ \\
$\mathbf{L i}$ & $16 \mathrm{c}$ & 0 & 0 & 0 & $0.50(1)$ & $0.83^{*}$ \\
$\mathbf{N i}$ & $16 \mathrm{c}$ & 0 & 0 & 0 & $0.50(1)$ & $0.06(4)$ \\
$\mathbf{O}$ & $32 \mathrm{e}$ & $0.2521(1)$ & $0.2521(1)$ & $0.2521(1)$ & 1 & $0.56^{*}$
\end{tabular}

${ }^{*}$ Fixed to the values refined from neutron diffraction data.

Table S4. Refined local cation ordered structure $\left(\mathrm{P4}_{1} 22\right)$ of $\mathrm{LiNiVO}_{4}$ using neutron PDF data $(1-8 \AA$, NOMAD, SNS)

\begin{tabular}{|c|c|c|c|c|c|c|}
\hline \multicolumn{7}{|c|}{ S.G. $P 4{ }_{1} 22 \quad a=5.8040(33) \AA, b=8.2828(91) \AA \quad R_{w p}=7.98 \%$} \\
\hline Atom & Wyck. & $x$ & $y$ & $z$ & Occ. & $B_{\text {iso }}\left(\AA^{2}\right)$ \\
\hline $\mathbf{V}$ & $4 \mathrm{c}$ & $0.2570(27)$ & $0.2570(27)$ & $3 / 8$ & 1 & $0.30^{*}$ \\
\hline Li1 & $4 \mathrm{~b}$ & $1 / 2$ & $0.2494(28)$ & 0 & $0.73(1)$ & $0.65(1)$ \\
\hline
\end{tabular}




\begin{tabular}{lllllll}
\hline Ni1 & $4 \mathrm{~b}$ & $1 / 2$ & $0.2494(28)$ & 0 & $0.27(1)$ & $0.32(1)$ \\
Li2 & $4 \mathrm{a}$ & 0 & $0.2490(17)$ & 0 & $0.27(1)$ & $0.65(1)$ \\
Ni2 & $4 \mathrm{a}$ & 0 & $0.2490(17)$ & 0 & $0.73(1)$ & $0.32(1)$ \\
O1 & $8 \mathrm{~d}$ & $0.2416(13)$ & $-0.0095(8)$ & 0 & 1 & $0.48(1)$ \\
O2 & $8 \mathrm{~d}$ & $0.2438(13)$ & $0.4959(6)$ & $0.0088(6)$ & 1 & $0.48(1)$
\end{tabular}

*Fixed to the value refined using X-ray PDF.

Table S5. Refined local cation ordered structure $\left(\mathrm{P4}_{1} 22\right)$ of $\mathrm{LiNiVO}_{4}$ using X-ray PDF data (1-8 $\AA$, 1 1-IDB, APS)

\begin{tabular}{llccccl}
\hline \multicolumn{6}{c}{ S.G. $P 4_{1} 22$} & $\mathrm{a}=5.8063(49)$ \\
Atom & Wyck. & $x$ & $y$ & $z$ & Occ. & $B_{\text {iso }}\left(\AA^{2}\right)$ \\
V & $4 c$ & $0.2529(5)$ & $0.2529(5)$ & $3 / 8$ & 1 & $0.30(2)$ \\
Li1 & $4 b$ & $1 / 2$ & $0.2456(13)$ & 0 & $0.74(2)$ & $0.65^{*}$ \\
Ni1 & $4 b$ & $1 / 2$ & $0.2456(13)$ & 0 & $0.26(2)$ & $0.42(4)$ \\
Li2 & $4 a$ & 0 & $0.2381(20)$ & 0 & $0.26(2)$ & $0.65^{*}$ \\
Ni2 & $4 a$ & 0 & $0.2381(20)$ & 0 & $0.74(2)$ & $0.42(4)$ \\
O1 & $8 d$ & $0.2520(43)$ & $-0.0020(16)$ & 0 & 1 & $0.94(2)$ \\
O2 & $8 d$ & $0.2619(16)$ & $0.5199(16)$ & $-0.0021(11)$ & 1 & $0.94(2)$
\end{tabular}

${ }^{*}$ Fixed to the value refined using neutron PDF.

Table S6. Refined local cation ordered structure (Imma) of $\mathrm{LiNiVO}_{4}$ using neutron PDF data (1-8 , NOMAD, SNS)

\begin{tabular}{|c|c|c|c|c|c|c|}
\hline \multicolumn{7}{|c|}{ S.G. $\operatorname{Imma} a=5.7903(75) \AA \mathrm{b}=5.8186(89) \AA \mathrm{c}=8.2856(139) \AA \mathrm{R}_{\mathrm{wp}}=10.58 \%$} \\
\hline Atom & Wyck. & $x$ & $y$ & $z$ & Occ. & $B_{\text {iso }}\left(\AA^{2}\right)$ \\
\hline
\end{tabular}




\begin{tabular}{ccccccc}
\hline $\mathbf{V}$ & $4 \mathrm{e}$ & 0 & $1 / 4$ & $0.6188(51)$ & 1 & $0.30^{*}$ \\
Li1 & $4 \mathrm{c}$ & $1 / 4$ & $1 / 4$ & $1 / 4$ & $0.59(3)$ & $1.60(1)$ \\
Ni1 & $4 \mathrm{c}$ & $1 / 4$ & $1 / 4$ & $1 / 4$ & $0.41(3)$ & $0.40(1)$ \\
$\mathbf{L i 2}$ & $4 \mathrm{a}$ & 0 & 0 & 0 & $0.41(3)$ & $1.60(1)$ \\
Ni2 & $4 \mathrm{a}$ & 0 & 0 & 0 & $0.59(3)$ & $0.40(1)$ \\
O1 & $8 \mathrm{~h}$ & 0 & $0.5048(11)$ & $0.2482(19)$ & 1 & $0.56(3)$ \\
O2 & $8 \mathrm{i}$ & $0.2465(18)$ & $1 / 4$ & $-0.0095(8)$ & 1 & $0.48(1)$
\end{tabular}

${ }^{*}$ Fixed to the value refined using X-ray PDF.

Table S7. Refined long range structure (Fd-3m) of $\mathrm{LiCoVO}_{4}$ (POWGEN, SNS)

\begin{tabular}{|c|c|c|c|c|c|c|}
\hline & \multicolumn{3}{|c|}{ S.G.Fd-3m a $=8.21247(3) \AA$} & \multicolumn{2}{|c|}{$\mathrm{R}_{\mathrm{wp}}=2.94 \% \chi^{2}=1.94$} & \\
\hline Atom & Wyck. & $x$ & $y$ & $z$ & Occ. & $B_{\text {iso }}\left(\AA^{2}\right)$ \\
\hline $\mathbf{V}$ & $8 b$ & 0.375 & 0.375 & 0.375 & 1 & 0.4 \\
\hline${ }^{7} \mathbf{L i}$ & $16 c$ & 0 & 0 & 0 & $0.49(1)$ & $0.8318)$ \\
\hline Co & $16 c$ & 0 & 0 & 0 & $0.51(1)$ & $0.24(5)$ \\
\hline $\mathbf{O}$ & $32 \mathrm{e}$ & $0.2529(1)$ & $0.2529(1)$ & $0.2529(1)$ & 1 & $0.56(1)$ \\
\hline
\end{tabular}

Table S8. Refined local cation ordered structure $\left(\mathrm{P4}_{1} 22\right)$ of $\mathrm{LiCoVO}_{4}$ using neutron PDF data $(1-8 \AA$, NOMAD, SNS)

\begin{tabular}{llcccll}
\hline \multicolumn{6}{l}{ S.G. $P 4{ }_{1} 22$} & $\mathrm{a}=5.8575(56) \AA \mathrm{b}=8.3039(149) \AA \mathrm{R}_{\mathrm{wp}}=9.98 \%$ \\
\hline Atom & Wyck. & $x$ & $y$ & $z$ & Occ. & $B_{\text {iso }}\left(\AA^{2}\right)$ \\
V & $4 \mathrm{c}$ & $0.2499(46)$ & $0.2499(46)$ & $3 / 8$ & 1 & 0.30 \\
Li1 & $4 \mathrm{~b}$ & $1 / 2$ & $0.2854(28)$ & 0 & $0.33(2)$ & $0.66(1)$ \\
Co1 & $4 \mathrm{~b}$ & $1 / 2$ & $0.2854(28)$ & 0 & $0.67(2)$ & $0.32(1)$ \\
Li2 & $4 \mathrm{a}$ & 0 & $0.2396(20)$ & 0 & $0.67(2)$ & $0.66(1)$ \\
Co2 & $4 \mathrm{a}$ & 0 & $0.2396(20)$ & 0 & $0.33(2)$ & $0.32(1)$ \\
$\mathbf{O 1}$ & $8 \mathrm{~d}$ & $0.2438(9)$ & $-0.0146(5)$ & 0 & 1 & $0.48(1)$ \\
O2 & $8 \mathrm{~d}$ & $0.2556(9)$ & $0.5019(5)$ & $0.0066(6)$ & 1 & $0.48(1)$ \\
\hline
\end{tabular}

Table S9. Refined long range structure (Fd-3m) of $\mathrm{NiAl}_{2} \mathrm{O}_{4}$ (NOMAD, SNS)

$$
\text { S.G. Fd-3m a }=8.0538(1) \AA \quad \mathrm{R}_{\mathrm{wp}}=4.39 \% \chi^{2}=3.36
$$




\begin{tabular}{lllllll}
\hline Atom & Wyck. & \multicolumn{1}{c}{$x$} & \multicolumn{1}{c}{$y$} & \multicolumn{1}{c}{$z$} & Occ. & $B_{\text {iso }}\left(\AA^{2}\right)$ \\
Al & $8 \mathrm{~b}$ & 0.375 & 0.375 & 0.375 & 1 & $0.31(1)$ \\
$\mathrm{Ni}$ & $16 \mathrm{c}$ & 0 & 0 & 0 & $0.61(1)$ & $0.30(1)$ \\
$\mathrm{Al}$ & $16 \mathrm{c}$ & 0 & 0 & 0 & $0.38(1)$ & $0.30(1)$ \\
$\mathbf{O}$ & $32 \mathrm{e}$ & $0.2456(1)$ & $0.2456(1)$ & $0.2456(1)$ & 1 & $1.06(1)$ \\
\hline
\end{tabular}

Table S10. Refined local cation ordered structure $\left(\mathrm{P4}_{1} 22\right)$ of $\mathrm{NiAl}_{2} \mathrm{O}_{4}$ using neutron PDF data (1-8 $\AA$, NOMAD, SNS)

\begin{tabular}{|c|c|c|c|c|c|c|}
\hline & \multicolumn{6}{|c|}{ S.G.P4 $22 \quad a=5.6812(20) \AA \quad b=8.1338(67) \AA R_{w p}=9.28 \%$} \\
\hline Atom & Wyck. & $x$ & $y$ & $z$ & Occ. & $B_{\text {iso }}\left(\AA^{2}\right)$ \\
\hline Al1 & $4 \mathrm{c}$ & $0.2537(5)$ & $0.2537(5)$ & $3 / 8$ & 1 & $0.31(1)$ \\
\hline Nil & $4 \mathrm{~b}$ & $1 / 2$ & $0.2442(5)$ & 0 & $0.00(2)$ & $0.30(1)$ \\
\hline Al2 & $4 \mathrm{~b}$ & $1 / 2$ & $0.2442(5)$ & 0 & $1.00(2)$ & $0.30(1)$ \\
\hline Ni2 & $4 a$ & 0 & $0.2451(9)$ & 0 & $1.00(2)$ & $0.51(2)$ \\
\hline Al3 & $4 a$ & 0 & $0.2451(9)$ & 0 & $0.00(2)$ & $0.51(2)$ \\
\hline $\mathbf{O 1}$ & $8 \mathrm{~d}$ & $0.2645(9)$ & $0.0014(5)$ & 0 & 1 & $0.60(1)$ \\
\hline $\mathbf{O 2}$ & $8 \mathrm{~d}$ & $0.2604(11)$ & $0.4918(11)$ & $-0.0171(7)$ & 1 & $0.60(1)$ \\
\hline
\end{tabular}

Table S1 1. Refined long range structure $(\mathrm{Fd}-3 \mathrm{~m})$ of $\mathrm{MgIn}_{2} \mathrm{O}_{4}(\mathrm{Cu} \mathrm{K \alpha})$

\begin{tabular}{|c|c|c|c|c|c|c|}
\hline & \multicolumn{3}{|c|}{ S.G.Fd-3m a = 8.21247(3) $\AA$} & \multicolumn{2}{|c|}{$\mathrm{R}_{\mathrm{wp}}=2.94 \% \chi^{2}=1.94$} & \\
\hline Atom & Wyck. & $x$ & $y$ & $z$ & Occ. & $B_{\text {iso }}\left(\AA^{2}\right)$ \\
\hline In1 & $8 b$ & 0.375 & 0.375 & 0.375 & 1 & 0.4 \\
\hline Mg & $16 c$ & 0 & 0 & 0 & $0.49(1)$ & $0.81(8)$ \\
\hline In2 & $16 \mathrm{c}$ & 0 & 0 & 0 & $0.51(1)$ & $0.24(5)$ \\
\hline $\mathbf{O}$ & $32 \mathrm{e}$ & $0.2529(1)$ & $0.2529(1)$ & $0.2529(1)$ & 1 & $0.56(1)$ \\
\hline
\end{tabular}


Table S12. Refined local cation ordered structure $\left(\mathrm{P4}_{1} 22\right)$ of $\mathrm{MgIn}_{2} \mathrm{O}_{4}$ using X-ray PDF data (1-8 , NOMAD, SNS)

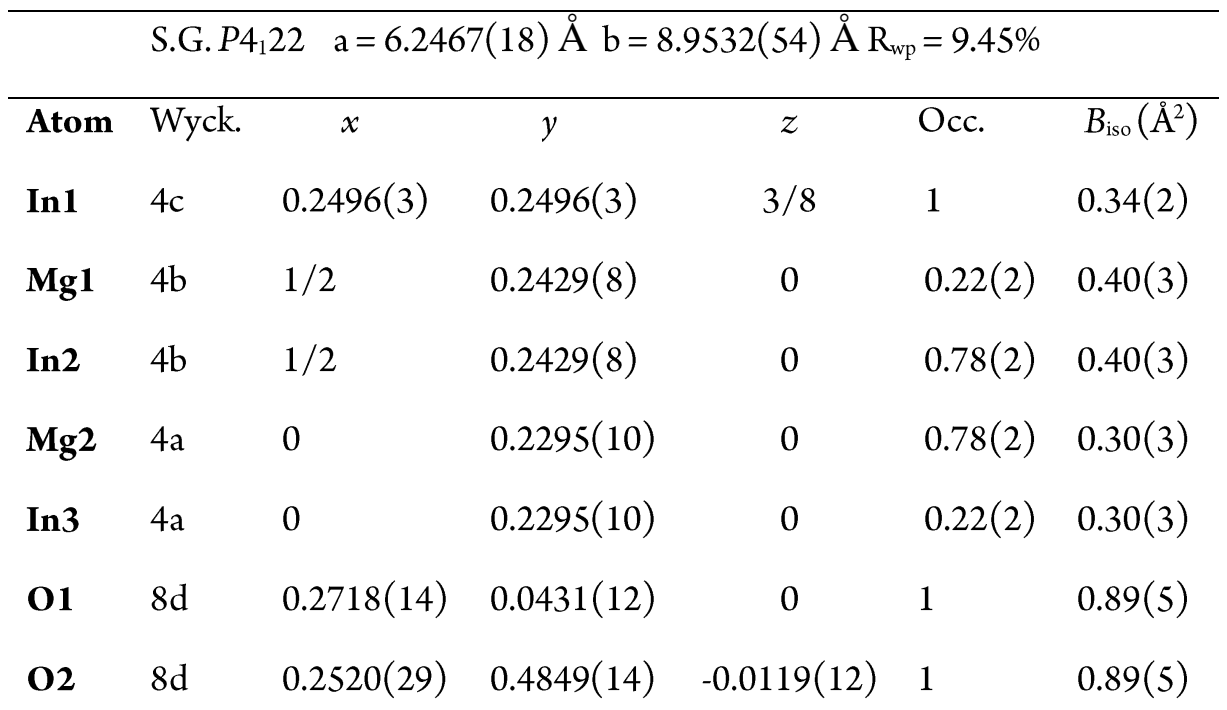

Table S13. Refined long range structure (Fd-3m) of $\mathrm{LiCrTiO}_{4}$ (POWGEN, SNS)

\begin{tabular}{|c|c|c|c|c|c|c|}
\hline & \multicolumn{3}{|c|}{ S.G. Fd-3m a = 8.31101(5) $\AA$} & \multicolumn{2}{|c|}{$\mathrm{R}_{\mathrm{wp}}=2.89 \% \chi^{2}=1.55$} & \\
\hline Atom & Wyck. & $x$ & $y$ & $z$ & Occ. & $B_{\text {iso }}\left(\AA^{2}\right)$ \\
\hline${ }^{7} \mathbf{L i}$ & $8 b$ & 0.375 & 0.375 & 0.375 & 1 & $0.75(1)$ \\
\hline $\mathrm{Cr}$ & $16 c$ & 0 & 0 & 0 & $0.50(1)$ & $0.21(10)$ \\
\hline $\mathbf{T i}$ & $16 c$ & 0 & 0 & 0 & $0.50(1)$ & $0.21(10)$ \\
\hline $\mathbf{O}$ & $32 \mathrm{e}$ & $0.2375(1)$ & $0.2375(1)$ & $0.2375(1)$ & 1 & $0.37(1)$ \\
\hline
\end{tabular}

Table S14. Refined local cation ordered structure $\left(\mathrm{P4}_{1} 22\right)$ of $\mathrm{LiCrTiO}_{4}$ using neutron $\mathrm{PDF}$ data $(1-8 \AA$, NOMAD, SNS)

\begin{tabular}{|c|c|c|c|c|c|c|}
\hline \multicolumn{7}{|c|}{ S.G.P4 $22 \quad a=5.8947(16) \quad b=8.2926(40) \mathrm{R}_{w p}=7.41 \%$} \\
\hline Atom & Wyck. & $x$ & $y$ & $z$ & Occ. & $B_{\text {iso }}\left(\AA^{2}\right)$ \\
\hline${ }^{7} \mathbf{L i}$ & $4 c$ & $0.2350(7)$ & $0.2350(7)$ & $3 / 8$ & 1 & $1.13(6)$ \\
\hline Cr1 & $4 \mathrm{~b}$ & $1 / 2$ & $0.2325(17)$ & 0 & $0.32(1)$ & $0.20(1)$ \\
\hline Til & $4 \mathrm{~b}$ & $1 / 2$ & $0.2325(17)$ & 0 & $0.68(1)$ & $0.20(1)$ \\
\hline Cr2 & $4 a$ & 0 & $0.2403(15)$ & 0 & $0.68(1)$ & $0.31(1)$ \\
\hline Ti2 & $4 a$ & 0 & $0.2403(15)$ & 0 & $0.32(1)$ & $0.31(1)$ \\
\hline
\end{tabular}




\begin{tabular}{lllllll}
\hline $\mathbf{O 1}$ & $8 \mathrm{~d}$ & $0.2601(5)$ & $0.0313(4)$ & $-0.0044(3)$ & 1 & $0.59(2)$ \\
$\mathbf{O 2}$ & $8 \mathrm{~d}$ & $0.2518(4)$ & $0.4739(4)$ & $-0.0122(2)$ & 1 & $0.59(2)$
\end{tabular}

Table S1 5. Refined long range structure (Fd-3m) of $\mathrm{Zn}_{2} \mathrm{TiO}_{4}(\mathrm{NOMAD}, \mathrm{SNS})$

\begin{tabular}{|c|c|c|c|c|c|c|}
\hline & \multicolumn{3}{|c|}{ S.G. Fd-3m a $=8.4747(3) \AA$} & \multicolumn{2}{|c|}{$\mathrm{R}_{\mathrm{wp}}=3.44 \% \chi^{2}=3.71$} & \\
\hline Atom & Wyck. & $x$ & $y$ & $z$ & Occ. & $B_{\text {iso }}\left(\AA^{2}\right)$ \\
\hline Zn1 & $8 b$ & 0.375 & 0.375 & 0.375 & 1 & $0.28(3)$ \\
\hline $\mathbf{T i}$ & $16 c$ & 0 & 0 & 0 & $0.48(2)$ & $0.55(2)$ \\
\hline Zn2 & $16 c$ & 0 & 0 & 0 & $0.52(1)$ & $0.55(2)$ \\
\hline $\mathbf{O}$ & $32 \mathrm{e}$ & $0.2399(1)$ & $0.2399(1)$ & $0.2399(1)$ & 1 & $1.04(1)$ \\
\hline
\end{tabular}

Table S16. Refined local cation ordered structure $\left(\mathrm{P4}_{1} 22\right)$ of $\mathrm{Zn}_{2} \mathrm{TiO}_{4}$ using neutron PDF data $(1-8 \AA$, NOMAD, SNS)

\begin{tabular}{lllcccc}
\hline \multicolumn{6}{c}{ S.G. $P 4{ }_{1} 22$} & $\mathrm{a}=5.9966(28)$ \\
Atom & Wyck. & $x$ & $y$ & $z$ & Occ. & $B_{\text {iso }}\left(\AA^{2}\right)$ \\
Zn & $4 c$ & $0.2466(6)$ & $0.2466(6)$ & $3 / 8$ & 1 & $0.44(5)$ \\
Zn1 & $4 b$ & $1 / 2$ & $0.2870(12)$ & 0 & $0.99(2)$ & $0.30(7)$ \\
Ti1 & $4 b$ & $1 / 2$ & $0.2870(12)$ & 0 & $0.01(2)$ & $0.30(7)$ \\
Zn2 & $4 \mathrm{a}$ & 0 & $0.2693(11)$ & 0 & $0.01(2)$ & $0.80(8)$ \\
Ti2 & $4 \mathrm{a}$ & 0 & $0.2693(11)$ & 0 & $0.99(2)$ & $0.80(8)$ \\
O1 & $8 \mathrm{~d}$ & $0.2595(6)$ & $0.0313(5)$ & $0.0028(5)$ & 1 & $0.62(4)$ \\
O2 & $8 \mathrm{~d}$ & $0.2628(7)$ & $0.4881(8)$ & $-0.0200(4)$ & 1 & $0.62(4)$ \\
\hline
\end{tabular}

Table S17. Refined long range structure (Fd-3m) of $\mathrm{Zn}_{2} \mathrm{SnO}_{4}$ (NOMAD, SNS)

\begin{tabular}{|c|c|c|c|c|c|c|}
\hline & \multicolumn{2}{|c|}{ S.G.Fd-3m } & $\mathrm{a}=8.6456(2) \AA$ & \multicolumn{2}{|c|}{$\mathrm{R}_{\mathrm{wp}}=4.43 \% \chi^{2}=3.76$} & \\
\hline Atom & Wyck. & $x$ & $y$ & $z$ & Occ. & $B_{\text {iso }}\left(\AA^{2}\right)$ \\
\hline Zn1 & $8 b$ & 0.37 & 0.375 & 0.375 & 1 & $0.48(1)$ \\
\hline Sn & $16 \mathrm{c}$ & 0 & 0 & 0 & $0.48(2)$ & $0.86(2)$ \\
\hline $\operatorname{Zn} 2$ & $16 c$ & 0 & 0 & 0 & $0.52(2)$ & $0.86(2)$ \\
\hline
\end{tabular}




\begin{tabular}{lllllll}
\hline O & $32 \mathrm{e}$ & $0.2416(1)$ & $0.2416(1)$ & $0.2416(1)$ & 1 & $0.56(1)$
\end{tabular}

Table S18. Refined local cation ordered structure $\left(P_{4}{ }_{12}\right)$ of $\mathrm{Zn}_{2} \mathrm{SnO}_{4}$ using neutron PDF data (1-8 , NOMAD, SNS)

\begin{tabular}{|c|c|c|c|c|c|c|}
\hline & \multicolumn{6}{|c|}{ S.G.P4 ${ }_{1} 22 a=6.1221(46) \AA \mathrm{A}=8.6846(127) \AA \mathrm{R}_{\mathrm{wp}}=9.35 \%$} \\
\hline Atom & Wyck. & $x$ & $y$ & $z$ & Occ. & $B_{\text {iso }}\left(\AA^{2}\right)$ \\
\hline Zn1 & $4 \mathrm{c}$ & $0.2487(1)$ & $0.2487(1)$ & $3 / 8$ & 1 & $0.63(2)$ \\
\hline Zn2 & $4 \mathrm{~b}$ & $1 / 2$ & $0.2428(2)$ & 0 & $0.99(12)$ & $0.52(4)$ \\
\hline Sn 1 & $4 \mathrm{~b}$ & $1 / 2$ & $0.2428(2)$ & 0 & $0.01(12)$ & $0.52(4)$ \\
\hline Zn3 & $4 a$ & 0 & $0.2295(9)$ & 0 & $0.99(12)$ & $0.54(4)$ \\
\hline Sn2 & $4 a$ & 0 & $0.2295(9)$ & 0 & $0.01(12)$ & $0.54(4)$ \\
\hline O1 & $8 \mathrm{~d}$ & $0.2604(10)$ & $0.0143(8)$ & $-0.0051(6)$ & 1 & $0.74(2)$ \\
\hline $\mathbf{O 2}$ & $8 \mathrm{~d}$ & $0.2563(8)$ & $0.4846(10)$ & $-0.0141(8)$ & 1 & $0.74(2)$ \\
\hline
\end{tabular}

Table S19. Refined local cation ordered structure $\left(\mathrm{P4}_{1} 22\right)$ of $\mathrm{Zn}_{2} \mathrm{SnO}_{4}$ using X-ray PDF data (1-8 $\AA$, 1 1-IDB, APS)

\begin{tabular}{lllcccc}
\hline \multicolumn{6}{c}{ S.G. $P 4_{1} 22$} & $\mathrm{a}=6.1270(28) \AA \mathrm{b}=8.7262(86) \AA \mathrm{R}_{\mathrm{wp}}=10.17 \%$ \\
\hline Atom & Wyck. & $x$ & $y$ & $z$ & Occ. & $B_{\text {iso }}\left(\AA^{2}\right)$ \\
$\mathbf{Z n 1}$ & $4 \mathrm{c}$ & $0.2498(3)$ & $0.2498(3)$ & $3 / 8$ & 1 & $0.45(4)$ \\
$\mathbf{Z n 2}$ & $4 \mathrm{~b}$ & $1 / 2$ & $0.2390(6)$ & 0 & $1.00(3)$ & $0.54(2)$ \\
$\mathbf{S n 1}$ & $4 \mathrm{~b}$ & $1 / 2$ & $0.2390(6)$ & 0 & $0.00(3)$ & $0.54(2)$ \\
$\mathbf{Z n 3}$ & $4 \mathrm{a}$ & 0 & $0.2305(7)$ & 0 & $0.00(3)$ & $0.63(2)$ \\
Sn2 & $4 \mathrm{a}$ & 0 & $0.2305(7)$ & 0 & $1.00(3)$ & $0.63(2)$ \\
$\mathbf{O 1}$ & $8 \mathrm{~d}$ & $0.2569(55)$ & $-0.0023(14)$ & $0.0102(14)$ & 1 & $0.93(4)$ \\
$\mathbf{O 2}$ & $8 \mathrm{~d}$ & $0.2486(43)$ & $0.4594(24)$ & $-0.0263(5)$ & 1 & $0.93(4)$ \\
\hline
\end{tabular}


Table S20. Refined long range cation ordered structure $\left(\mathrm{P4}_{1} 22\right)$ of $\mathrm{LiZnNbO}_{4}$ using synchrotron $\mathrm{XRD}$ (11ID-B, APS, $\lambda=0.2113 \AA$ )

\begin{tabular}{|c|c|c|c|c|c|c|}
\hline \multicolumn{7}{|c|}{ S.G.P4122 a $=6.0810(1) \AA \quad b=8.3999(1) \AA \mathrm{R}_{\mathrm{wp}}=9.98 \%$} \\
\hline Atom & Wyck. & $x$ & $y$ & $z$ & Occ. & $B_{\text {iso }}\left(\AA^{2}\right)$ \\
\hline Zn & $4 c$ & $0.2598(2)$ & $0.2598(2)$ & $3 / 8$ & 1 & $0.20(3)$ \\
\hline Nb 1 & $4 \mathrm{~b}$ & $1 / 2$ & $0.2146(2)$ & 0 & 1 & $0.02(3)$ \\
\hline $\mathrm{Li2}$ & $4 a$ & 0 & $0.2075(32)$ & 0 & 1 & 1 \\
\hline 01 & $8 \mathrm{~d}$ & $0.2630(7)$ & $0.0168(7)$ & $0.0042(5)$ & 1 & $0.60(1)$ \\
\hline $\mathbf{O 2}$ & $8 \mathrm{~d}$ & $0.5190(7)$ & $0.2644(7)$ & $0.2328(5)$ & 1 & $0.60(1)$ \\
\hline
\end{tabular}

Table S21. The total energy of $\mathrm{V}[\mathrm{LiNi}] \mathrm{O}_{4}, \mathrm{Li}[\mathrm{CrTi}] \mathrm{O}_{4}, \mathrm{Al}[\mathrm{NiAl}] \mathrm{O}_{4}$ and $\mathrm{Sn}[\mathrm{ZnSn}] \mathrm{O}_{4}$ in three structural phases obtained by DFT calculation, the energy differences relative to the lowest ones are also listed.

\begin{tabular}{|c|c|c|c|c|}
\hline & Phase & $\mathrm{Fd}-3 \mathrm{~m}$ & Imma & $P 4_{1} 22$ \\
\hline $\mathrm{V}[\mathrm{LiNi}] \mathrm{O}_{4}$ & \multirow{4}{*}{$\begin{array}{c}\text { Total Energy (eV) } \\
\text { (Diff.) }\end{array}$} & $\begin{array}{l}-49.129 \\
(0.103) \\
\end{array}$ & $\begin{array}{r}-49.223 \\
(0.009) \\
\end{array}$ & $\begin{array}{r}-49.232 \\
(0.000) \\
\end{array}$ \\
\hline $\mathrm{Li}[\mathrm{CrTi}] \mathrm{O}_{4}$ & & $\begin{array}{l}-50.080 \\
(0.260) \\
\end{array}$ & $\begin{array}{l}-50.322 \\
(0.018) \\
\end{array}$ & $\begin{array}{l}-50.340 \\
(0.000) \\
\end{array}$ \\
\hline $\mathrm{Al}[\mathrm{NiAl}] \mathrm{O}_{4}$ & & $\begin{array}{r}-47.470 \\
(0.273) \\
\end{array}$ & $\begin{array}{r}-47.630 \\
(0.113) \\
\end{array}$ & $\begin{array}{r}-47.743 \\
(0.000) \\
\end{array}$ \\
\hline $\mathrm{Zn}[\mathrm{ZnSn}] \mathrm{O}_{4}$ & & $\begin{array}{l}-35.098 \\
(0.426)\end{array}$ & $\begin{array}{l}-35.430 \\
(0.094)\end{array}$ & $\begin{array}{l}-35.524 \\
(0.000)\end{array}$ \\
\hline
\end{tabular}

Table S22. Refined long range cation disordered structure $(\mathrm{Fd}-3 \mathrm{~m})$ of $\mathrm{LiMn}_{2} \mathrm{O}_{4}$ using neutron diffraction data (NOMAD, SNS, $300 \mathrm{~K}$ )

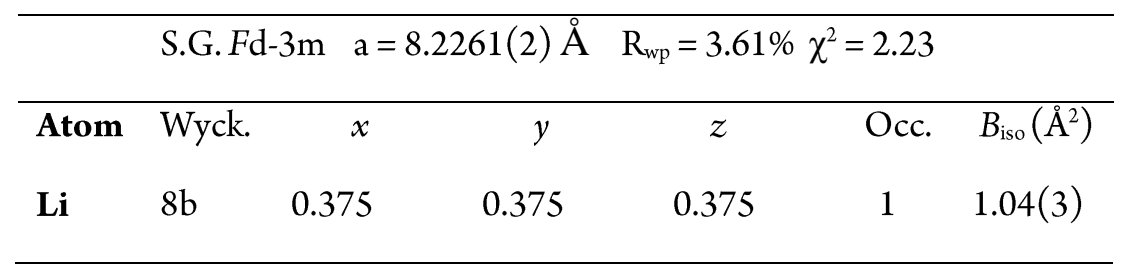




\begin{tabular}{lllllll}
\hline Mn & $16 \mathrm{c}$ & 0 & 0 & 0 & 1 & $0.84(1)$ \\
$\mathbf{O}$ & $32 \mathrm{e}$ & $0.2366(1)$ & $0.2366(1)$ & $0.2366(1)$ & 1 & $1.24(1)$ \\
\hline
\end{tabular}

Table S23. Refined short range cation ordered structure (Fddd) of $\mathrm{LiMn}_{2} \mathrm{O}_{4}$ using neutron PDF data (1-8 $\AA ̊$, NOMAD, SNS, 300 K)

\begin{tabular}{|c|c|c|c|c|c|c|}
\hline \multirow{2}{*}{$\begin{array}{c}\text { S.G. P4, } 22 \\
\text { Atom }\end{array}$} & \multicolumn{5}{|c|}{$a=8.1832(63) \AA \quad b=24.6686(194) \AA_{c}=24.9444(155) \AA$} & \multirow{2}{*}{$\begin{array}{c}\mathbf{R}_{\mathbf{w p}}=\mathbf{9 . 9 8} \% \\
B_{\text {iso }}\left(\AA^{2}\right)\end{array}$} \\
\hline & Wyck. & $x$ & $y$ & $z$ & Occ. & \\
\hline Lil & 8 & 0.125 & 0.125 & 0.125 & 1 & $0.98(5)$ \\
\hline Li2 & 16 & 0.125 & 0.125 & 0.4625 & 1 & $0.98(5)$ \\
\hline Li3 & 16 & 0.125 & 0.4557 & 0.125 & 1 & $0.98(5)$ \\
\hline Li4 & 32 & 0.3738 & 0.2096 & 0.0441 & 1 & $0.98(5)$ \\
\hline Mn1 & 16 & 0.5 & 0.5 & 0.5 & 1 & $0.23(1)$ \\
\hline Mn2 & 32 & 0.50078 & 0.0810 & 0.0852 & 1 & $0.23(1)$ \\
\hline Mn3 & 32 & 0.0003 & 0.1659 & 0.3303 & 1 & $0.23(1)$ \\
\hline Mn4 & 32 & 0.0059 & 0.1667 & 0.0058 & 1 & $0.23(1)$ \\
\hline Mn5 & 32 & 0.0045 & 0.2533 & 0.0820 & 1 & $0.23(1)$ \\
\hline 01 & 32 & 0.0182 & 0.3287 & 0.0819 & 1 & $0.69(1)$ \\
\hline $\mathbf{O 2}$ & 32 & 0.0154 & 0.0904 & 0.0061 & 1 & $0.69(1)$ \\
\hline $\mathbf{O 3}$ & 32 & 0.0115 & 0.2562 & 0.1598 & 1 & $0.69(1)$ \\
\hline 04 & 32 & 0.0148 & 0.0872 & 0.3233 & 1 & $0.69(1)$ \\
\hline 05 & 32 & 0.0229 & 0.0025 & 0.4225 & 1 & $0.69(1)$ \\
\hline 06 & 32 & 0.2387 & 0.0063 & 0.0068 & 1 & $0.69(1)$ \\
\hline $\mathbf{0 7}$ & 32 & 0.2618 & 0.0758 & 0.0821 & 1 & $0.69(1)$ \\
\hline 08 & 32 & 0.4798 & 0.0788 & 0.0078 & 1 & $0.69(1)$ \\
\hline 09 & 32 & 0.5160 & 0.0841 & 0.1614 & 1 & $0.69(1)$ \\
\hline
\end{tabular}


Table S24. Selected Mn bond lengths from the local cation ordered (S.G. Fddd) $\mathrm{LiMn}_{2} \mathrm{O}_{4}$ structure.

\begin{tabular}{|c|c|c|c|}
\hline Bond & Distance $(\AA ̊)$ & Bond & Distance $(\AA)$ \\
\hline Mn1-O5 (×2) & 1.8981 & $\mathrm{Mn} 4-\mathrm{O} 2$ & 1.8596 \\
\hline $\mathrm{Mn} 1-\mathrm{O} 8(\times 2)$ & 1.9516 & $\mathrm{Mn} 4-\mathrm{O} 2$ & 1.8641 \\
\hline Mn1-O6 (×2) & 2.1609 & Mn4-O6 & 1.8773 \\
\hline Mn2-O9 & 1.9103 & Mn4-O7 & 1.8891 \\
\hline Mn2-O9 & 1.9213 & Mn4-O8 & 1.9175 \\
\hline $\mathrm{Mn} 2-\mathrm{O} 8$ & 1.9561 & Mn4-O4 & 1.9674 \\
\hline Mn2-O7 & 1.9653 & Mn5-O1 & 1.8745 \\
\hline $\mathrm{Mn} 2-\mathrm{O} 5$ & 1.9970 & Mn5-O6 & 1.8934 \\
\hline Mn2-O9 & 2.1182 & Mn5-O5 & 1.9113 \\
\hline Mn3-O1 & 1.9348 & $\mathrm{Mn} 5-\mathrm{O} 3$ & 1.9136 \\
\hline Mn3-O4 & 1.9459 & $\mathrm{Mn} 5-\mathrm{O} 3$ & 1.9159 \\
\hline $\mathrm{Mn} 3-\mathrm{O} 3$ & 1.9727 & Mn5-O7 & 1.9486 \\
\hline $\mathrm{Mn} 3-\mathrm{O} 4$ & 1.9854 & & \\
\hline $\mathrm{Mn} 3-\mathrm{O} 2$ & 2.1619 & & \\
\hline Mn3-O1 & 2.2594 & & \\
\hline
\end{tabular}

1. Evjen, H., On the stability of certain heteropolar crystals. Physical Review 1932, 39 (4), 675. 